\title{
Gene expression profiles responses to aphid feeding in chrysanthemum (Chrysanthemum morifolium)
}

Xiaolong Xia ${ }^{\dagger}$, Yafeng Shao ${ }^{\dagger}$, Jiafu Jiang, Liping Ren, Fadi Chen, Weimin Fang, Zhiyong Guan and Sumei Chen ${ }^{*}$

\begin{abstract}
Background: Chrysanthemum is an important ornamental plant all over the world. It is easily attacked by aphid, Macrosiphoniella sanbourni. The molecular mechanisms of plant defense responses to aphid are only partially understood. Here, we investigate the gene expression changes in response to aphid feeding in chrysanthemum leaf by RNA-Seq technology.

Results: Three libraries were generated from pooled leaf tissues of Chrysanthemum morifolium 'nannongxunzhang' that were collected at different time points with $(Y)$ or without $(C K)$ aphid infestations and mock puncture treatment (Z), and sequenced using an Illumina HiSeq ${ }^{\text {TM }} 2000$ platform. A total of 7,363,292, 7,215,860 and 7,319,841 clean reads were obtained in library CK, $Y$ and $Z$, respectively. The proportion of clean reads was $>97.29 \%$ in each library. Approximately $76.35 \%$ of the clean reads were mapped to a reference gene database including all known chrysanthemum unigene sequences. 1,157, 527 and 340 differentially expressed genes (DEGs) were identified in the comparison of CK-VS-Y, CK-VS-Z and Z-VS-Y, respectively. These DEGs were involved in phytohormone signaling, cell wall biosynthesis, photosynthesis, reactive oxygen species (ROS) pathway and transcription factor regulatory networks, and so on.
\end{abstract}

Conclusions: Changes in gene expression induced by aphid feeding are shown to be multifaceted. There are various forms of crosstalk between different pathways those genes belonging to, which would allow plants to fine-tune its defense responses.

Keywords: Chrysanthemum, Aphid, Gene expression, RNA-Seq

\section{Background}

Chrysanthemum (Chrysanthemum morifolium Ramat.) is an important ornamental plants with high economic value all around the world [1,2]. It is susceptible to the aphid (Macrosiphoniella sanbourni) infestation from vegetative to flowering stage. $M$. sanbourni not only hampers the vegetative growth, but also decreases the quality of flowers, which causes serious loss in chrysanthemum production. Besides draining plants' nutrients, aphids also transmit pathogenic viruses. The progress has been made in our understanding of plant-aphid interaction, especially the molecular bases of plant resistance and defense against aphid feeding.

\footnotetext{
* Correspondence: chensm@njau.edu.cn

${ }^{\dagger}$ Equal contributors

College of Horticulture, Nanjing Agricultural University, No.1 Weigang, Nanjing 210095, China
}

Several plant $R$ (resistance) genes related with plant resistance to aphids have been identified. For example, Mi-1.2 gene confers resistance to the potato aphid (Macrosiphum euphorbiae Thomas) in wild tomato, Lycopersicon peruvianum (L.) P. Mill. [3,4]. And Vat (virus aphid transmission) gene mediates resistance to the cotton aphid (Aphis gossypii Glover) as well as some viruses transmitted by this aphid in melon, Cucumis melo L. [5]. Both Mi-1.2 and Vat gene belong to the nucleotide-binding-site leucine-rich-repeat (NBS-LRR) family of plant $R$ gene, and there is close linkage between resistance loci and NBS-LRR gene sequences revealed by genetic analysis [6,7]. However, detailed mechanisms of $R$ genes involved in aphid resistance still need further investigation.

During aphid infestation, series of plant defense responses, including plant hormone signal transduction, transcriptional 
regulation and the expression of defensive genes, would be induced [8]. Salicylic acid (SA), jasmonic acid (JA) and ethylene (ET) are three better studied phytohormones involved in aphid-induced plant hormone signal transduction. In interactions between Myzus persicae and Arabidopsis thaliana, SA signaling pathway is activated and the expression of genes such as pathogenesis-related (PR) genes (i.e., $\beta$-1,3-glucanase and chitinases) associated with the signaling pathway increased [9]. And a wide range of defensive responses in Arabidopsis thaliana attacked by cabbage aphid (Brevicoryne brassicae) depended on SA signaling [10]. Furthermore, recent studies found that SA signaling pathway was critical for $\mathrm{Mi}$-1.2-mediated resistance to aphid [11]. The JA pathway, containing wound hormone JA-Ile, is also an important regulator of plant resistance to herbivores. Kusnierczyk et al. [12] indicated that many of defense-associated responses induced by cabbage aphid (Brevicoryne brassicae) in wild-type (WT) plants were impaired in Arabidopsis lacking jasmonates. JA promoted the synthesis of glucosinolate and $\mathrm{N}^{\delta}$-acetyl ornithine in Arabidopsis, thereby improving the resistance to aphid $[13,14]$. The resistance of alfalfa to blue green aphid (BGA) and defense of Arabidopsis on cabbage aphid are all dependent on the JA signaling cascades [10,15]. Argandona and co-workers [16] observed that aphid feeding significantly induced the production of ET in the leaf tissue of aphid-resistant barley cultivars contrasted to susceptible ones. ET excited by green peach aphid infestation induced the expression of $A t M Y B 44$, which then bound to the promoter of ETHYLENE INSENSITIVE2 (EIN2), regulating the defense responses in Arabidopsis [17]. JA and ET often act synergistically, and are frequently antagonized by SA [18]. EIN2 is a bifunctional transducer of ET and JA signal transduction [8]. Still, knowledge about members of the EIN2 downstream defense pathway is limited.

The development of high-throughput technologies allows us a global view of gene expression changes during plant interactions with aphids. Moran et al. [19] suggested that genes associated with signaling, pathogenesis-related responses, oxidative stress and calcium-dependent signaling are crucial components of the aphid response profile in A. thaliana. Transcriptome and metabolome changes of Arabidopsis were investigated at $6,12,24$ and $48 \mathrm{~h}$ after $B$. brassicae infestation to monitor the progress of early response by full-genome oligonucleotide microarrays, revealing reactive oxygen species (ROS) and calcium is involved in early signaling, JA and SA in the regulation of defense responses, and the induction of transcripts associated with senescence, biosynthesis of indolyl glucosinolates (IGS), anti-insect proteins, camalexin, and several WRKY transcription factors were identified as well [10]. Kusnierczyk and co-workers [12] conducted an extensive analysis of transcriptional patterns of WT, aos defective in JA production, and fou 2 constitutively inducing JA biosynthesis in Arabidopsis. More than 200 genes whose expression were dependent on jasmonate levels and over 800 genes that differentially responded to aphid feeding in aos and fou 2 plants than in WT were identified through microarray. They also demonstrated activation of defense caused by JA, such as WRKY, ethylene responsive transcription factors (ERFs), BTB and TAZ domain protein 5 (BT5), pathogenesis related proteins $P R 1$ and $P R 2$, and plant defensines (PDFs). Numerous key genes and proteins were unravelled in researches of gene transcriptional responses in model plants, such as $A$. thaliana, Medicago truncatula, Nicotiana attenuata and Sorghum bicolor. However, the exact mechanisms and functions of most of them are still unclear.

Previous studies on chrysanthemum found that superoxide dismutase (SOD), peroxidase (POD), ascorbate peroxidase (APX), polyphenol oxidase (PPO) activity and phenylalanine ammonia lyase (PAL) activities were enhanced by aphid herbivory, and changes in the enzymes activities in resistant species were faster than those in susceptible ones [20]. SA and MeJA pretreatment improved the resistance of chrysanthemum against aphids and increased the content of ROS species, defensive substances, flavonoids and lignins (data not shown), which implied that multiple pathways should be involved in the response of chrysanthemum to the aphid infestation. Therefore, to make a comprehensive view of differentially expressed genes (DEGs) during chrysanthemum-Macrosiphoniella sanbourni interaction, an experiment exploring comparative expression profiling was conducted. We also conduct a mock puncture treatment which is designed to partially simulate the mechanical stress resulting from aphid penetration, attempting to figure out the potential impacts of aphid stylets. This work would lay a foundation for further study in the resistance of chrysanthemum to aphid.

\section{Results}

An overview of three libraries data sets by RNA-Seq

Three libraries were generated from pooled leaf tissues of Chrysanthemum morifolium 'nannongxunzhang' that were collected at different time points with (Y) or without (CK) aphid infestations and mock puncture treatment (Z), and sequenced using an Illumina HiSeq ${ }^{\mathrm{TM}} 2000$ platform. After removing reads containing adaptor sequence and with low-quality, a total of 7,363,292, 7,215,860 and $7,319,841$ clean reads were obtained, in library CK, Y and Z, respectively, corresponding to $360,801,308$, $353,577,140$ and 358,672,209 base pairs (Table 1) (Accession number SRS619289 for library CK; Accession number SRS627943 for library Y; Accession number SRS627944 for library Z). The proportion of clean reads was $>97.29 \%$ in each library (Additional file 1: Figure S1). And these clean reads were deposited in the NCBI Sequence Read 
Table 1 An overview of read mapping

\begin{tabular}{ccccccccc}
\hline Sample ID & Clean reads & $\begin{array}{c}\text { Total base } \\
\text { pairs }\end{array}$ & $\begin{array}{c}\text { Total mapped } \\
\text { reads }\end{array}$ & $\begin{array}{c}\text { Perfect } \\
\text { match }\end{array}$ & $\begin{array}{c}<=2 \text { bp } \\
\text { mismatch }\end{array}$ & $\begin{array}{c}\text { Unique } \\
\text { match }\end{array}$ & $\begin{array}{c}\text { Multi-position } \\
\text { match }\end{array}$ & $\begin{array}{c}\text { Total unmapped } \\
\text { reads }\end{array}$ \\
\hline CK & $7,363,292$ & $360,801,308$ & $5,678,491$ & $3,591,545$ & $2,086,946$ & $3,743,517$ & $1,934,974$ & $1,684,801$ \\
& $(100.00 \%)$ & $(100.00 \%)$ & $(77.12 \%)$ & $(48.78 \%)$ & $(28.34 \%)$ & $(50.84 \%)$ & $(26.28 \%)$ & $(22.88 \%)$ \\
Y & $7,215,860$ & $353,577,140$ & $5,444,023$ & $3,385,348$ & $2,058,675$ & $3,632,669$ & $1,811,354$ & $1,771,837$ \\
& $(100.00 \%)$ & $(100.00 \%)$ & $(75.45 \%)$ & $(46.92 \%)$ & $(28.53 \%)$ & $(50.34 \%)$ & $(25.10 \%)$ & $(24.55 \%)$ \\
\multirow{2}{*}{$Z$} & $7,319,841$ & $358,672,209$ & $5,598,453$ & $3,510,566$ & $2,087,887$ & $3,699,741$ & $1,898,712$ & $1,721,388$ \\
& $(100.00 \%)$ & $(100.00 \%)$ & $(76.48 \%)$ & $(47.96 \%)$ & $(28.52 \%)$ & $(50.54 \%)$ & $(25.94 \%)$ & $(23.52 \%)$ \\
\hline
\end{tabular}

CK: control; Y: aphid infestation treatment; Z: mock puncture treatment.

Archive database (http://trace.ncbi.nlm.nih.gov/Traces/ sra_sub/sub.cgi?) under accession number SRP042216.

A reference gene database including all known Chrysanthemum morifolium unigene sequences was applied to map the clean reads. According to the chosen criteria, an average of $76.35 \%$ of the clean reads were mapped (Table 1), which consisted of perfect match and $<=2$ bp mismatch. Regarding each library, the scales of clean reads uniquely mapped to the database were $50.84 \%, 50.34 \%$ and $50.54 \%$, respectively. There were still approximately $23.65 \%$ of clean reads that cannot be mapped, mainly due to the restriction of the reference gene database of chrysanthemum. The number of genes identified increased with the number of reads until above 6,000,000, implying saturation of sequencing (Figure 1). The unigene coverage analysed as a means of evaluating the quality of the RNA-Seq data was mostly $>50 \%$ (Figure 2 ).

\section{Differential expression and Gene ontology (GO) functional classification}

In library CK, there are 52,266 genes detected, and 50,894 and 51,631 genes in library $\mathrm{Y}$ and $\mathrm{Z}$, respectively. Among them, 2,656, 2,161 and 2,403 genes were specifically expressed in library CK, Y and Z, respectively; 46,507, 46,125 and 47,002 genes were co-expressed in library CK and $\mathrm{Y}$, library $\mathrm{Y}$ and $\mathrm{Z}$ or library $\mathrm{CK}$ and $\mathrm{Z}$, respectively; and 43,899 genes were simultaneously expressed in library $\mathrm{CK}, \mathrm{Y}$ and $\mathrm{Z}$ (Figure 3).

The transcript abundance of each gene was estimated by reads per kb per million reads (RPKM). Differentially expressed genes (DEGs) (Additional file 2: Table S1, Additional file 3: Table S2 and Additional file 4: Table S3) were identified according to Audic et al. [21], briefly $P$-value $<0.05, \quad F D R \leq 0.001$, and estimated absolute $\left|\log _{2} \operatorname{Ratio}(\mathrm{Z} / \mathrm{CK})\right| \geq 1$. Comparing the library CK with the library Y (CK-VS-Y), there were 1157 DEGs (995 genes up-regulated and 162 genes down-regulated, 995/162), and 527 (487/40) and 340 (213/127) DEGs in CK-VS-Z and Z-VS-Y, respectively (Figure 4A), of which 648, 143 and 76 genes were specifically expressed in CKVS-Y, CK-VS-Z and Z-VS-Y, respectively; 328, 83 and 208 genes were co-expressed in CK-VS-Y and CK-VS-Z, CK-VS-Z and Z-VS-Y or CK-VS-Y and Z-VS-Y, respectively; and 27 genes were simultaneously expressed in CK-VS-Y, CK-VS-Z and Z-VS-Y (Figure 4B).

For CK-VS-Y, 477 out of 1157 DEGs (477/1157) could be assigned a GO classification, and 210/527 and 134/340 for CK-VS-Z and Z-VS-Y, respectively (Additional file 5: Table S4, Additional file 6: Table S5 and Additional file 7: Table S6). In CK-VS-Y comparison, 336 DEGs were categorized as "biological process", 278 as "cellular component" and 378 as "molecular function" (336/278/378), and 136/121/159 and 97/72/100 in CK-VS-Z and Z-VS-Y,

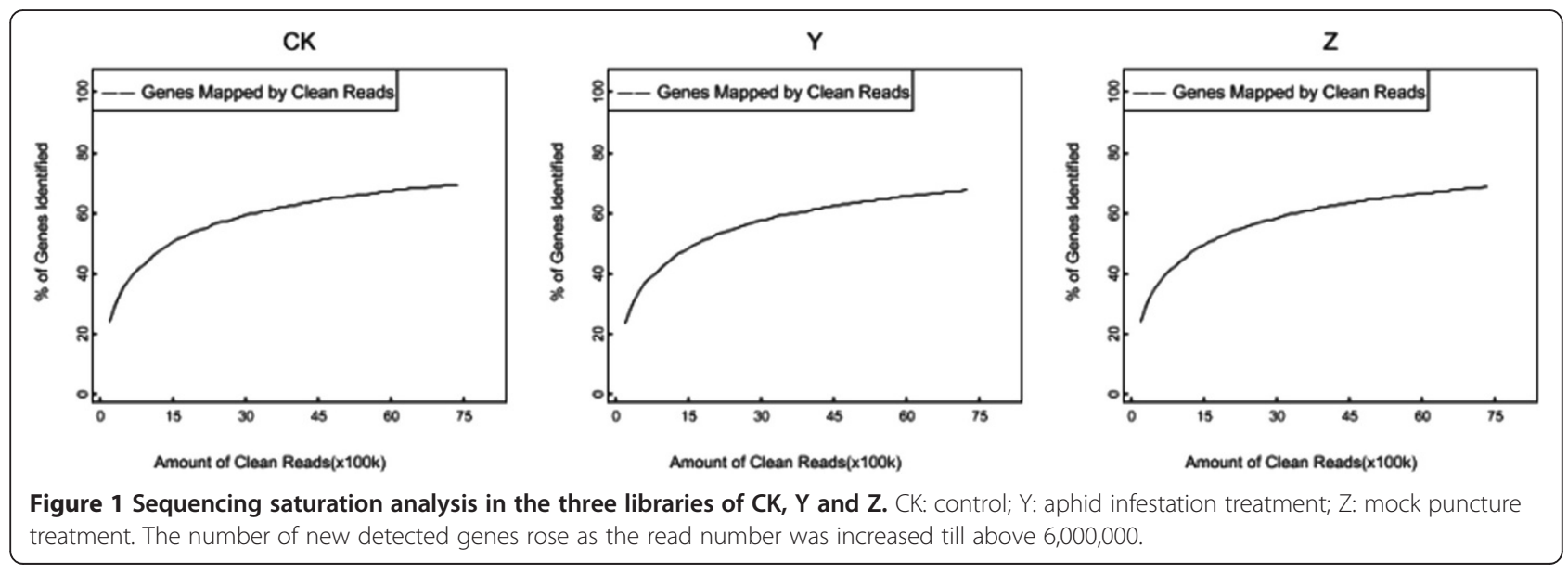






Figure 2 Distribution of gene coverage in each library (CK, Y and Z). CK: control; Y: aphid infestation treatment; Z: mock puncture treatment. The term "gene coverage" reflects the proportion of the full gene sequence represented by RNA-Seq reads.

respectively (Figure 5). The number of DEGs in most categories and categories of DEGs in the CK-VS-Y was higher than in the CK-VS-Z and Z-VS-Y, such as 'cell killing,' 'regulation of biological process', 'response to stimulus', 'signaling, and so on. Furthermore, in the CK-VS-Y (Figure 5A), most of DEGs were associated with cellular process, metabolic process and response to stimulus in terms of biological process, and in terms of cellular component, the majority were associated with cell, cell part, membrane and organelle, moreover, most were associated with binding and catalytic activity in terms of molecular function.

\section{Differentially expressed genes (DEGs) identified from aphid feeding and mock puncture treatments}

In this study, hundreds of DEGs involved in different pathways in response to aphid feeding (CK-VS-Y) and mock puncture treatments (CK-VS-Z) were recognized. Some of them responded to both aphid infestation and puncture treatments, such as NPR1, JAZ, MYC2 and DELLA involved in SA-JA-ET signaling network (Tables 2 and 3); WRKY, MYB and AP2/ERF transcription



Figure 3 The number of genes detected in library $C K, Y$ and $Z$. $C K$ : control; $Y$ : aphid infestation treatment; $Z$ : mock puncture treatment. factors (Tables 4 and 5); ROS scavenging enzymes (Additional file 8: Table S7 and Additional file 9: Table S8); COBRA-like and cellulose synthase like (Csl) genes participating in cell wall biosynthesis (Additional file 10: Table S9 and Additional file 11: Table S10); and terpene synthase encoding genes in secondary metabolism (Additional file 12: Table S11 and Additional file 13: Table S12). Whereas, several DEGs were specifically expressed in aphid treatment, such as NADPH oxidase genes (Additional file 8: Table S7) and photosynthesisrelated genes (Additional file 14: Table S13). Furthermore, individual members of a homologous gene family co-responding to aphid feeding and mock puncture treatment expressed preferentially either in CK-VS-Y or CK-VS-Z (Tables 2, 3, 4 and 5).

\section{Quantitative real-time PCR (qRT-PCR) validation of differentially expressed genes (DEGs) from RNA-Seq}

To validate the results of Illumina RNA-Seq, several genes from library CK and Y (CK: control; Y: aphid infestation treatment) were chosen randomly for qRT-PCR. For comparison of fold change between RNA-Seq and qRT-PCR, scatterplots were generated using the $\log _{2}$ fold change determined by RNA-Seq and qRT-PCR. As shown in Figure 6, the qRT-PCR results revealed that the expression tendency of these genes showed significant similarity $\left(r^{2}=0.92\right)$ with the Illumina RNA-Seq data, suggesting the reproducibility and accuracy of RNA-Seq results.

\section{Discussion}

RNA sequencing technology allows us to have a comprehensive view on the gene expression changes induced by aphids. And there are numerous genes whose expressions are changed after aphid feeding. Here, we mainly focus on the discussion on genes related to phytohormone signaling pathways and aphid feeding-associated transcription factors (TFs), photosynthesis, reactive oxygen species (ROS), cell wall biosynthesis and nucleotide-binding-site leucine-rich-repeat (NBS-LRR) genes. 
A

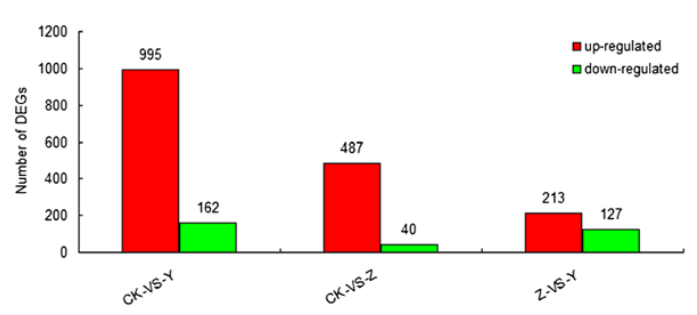

B



Figure 4 The number of differentially expressed genes (DEGs) identified in CK-VS-Y, CK-VS-Z and Z-VS-Y comparison. CK: control; Y: aphid infestation treatment; Z: mock puncture treatment. CK-VS-Y: comparison between CK and Y. CK-VS-Z: comparison between CK and Z. $Z$-VS-Y: comparison between $Z$ and $Y$. The criteria used for assigning significance were: $P$-value $<0.05, F D R \leq 0.001$, and estimated absolute $\mid \log _{2}$ Ratio(Y/CK) $\mid \geq 1$. A: number of DEGs up- or down-regulated in CK-VS-Y, CK-VS-Z and Z-VS-Y comparison; B: number of DEGs specifically or co-expressed in CK-VS-Y, CK-VS-Z and Z-VS-Y comparison.

\section{Plant hormone signaling pathway involved in plant-aphid interaction}

Salicylic acid (SA), jasmonic acid (JA) and ethylene (ET) are three major phytohormones reported in the regulation of signaling networks involved in aphid-induced defense responses. SA is important for localized plant tissue hypersensitive responses (HR), and could activate systemic acquired resistance (SAR), which is a broad-spectrum resistance of plants and it is necessary to transduce SA signal to stimulate the transcription of defense response genes, such as pathogenesis-related $(P R)$ genes [18,22,23]. Non-expressor of pathogenesis-related genes1 (NPR1), also called non-inducible immunity1 (NIM1), is a key factor of SAR, and activates the expression of $P R$ genes upon binding to TGAs, transcription factors which bind to SA-responsive elements (TGACG) in the promoters of $P R$ genes [24]. Besides its regulatory role in $P R$ gene expression, NPR1 also participates in the inhibition of JA signaling by SA [25]. In the present study, three NPR1 genes (Unigene107_All, Unigene23699_All and Unigene16290_All) and two TGA genes (Unigene2058_All and Unigene3706_All) were up-regulated by aphid infestation in the CK and Y comparison (CK-VS-Y) (Table 2). Two NPR1 genes (Unigene107_All and Unigene23699_All) was induced by mock puncture treatment in the CK and $\mathrm{Z}$ comparison (CK-VS-Z) (Table 3), implying that Unigene16290_All might respond specifically to aphid feeding. In Arabidopsis thaliana, npr1 and nim1 mutant plants are deficient in SA-induced disease resistance [24]. Further study suggests that the cytosolic function of NPR1 plays a role in SA-JA antagonism, and the nuclear function of NPR1 plays a role in the induction of SA-responsive genes $[25,26]$.

The JA signaling transduction, containing wound hormone jasmonoyl-isoleucine (JA-Ile), is another well studied regulator of plant resistance to aphids $[27,28]$. Genes involved in JA synthesis [29], such as phospholipase, lipoxygenase $(L O X)$, allene oxide synthase (AOS), allene oxide cyclase $(A O C)$ and 12-oxophytodienoic acid reductase
$(O P R)$ were all stimulated after aphid infestation and mock puncture treatment in the CK-VS-Y and CK-VS-Z (Tables 2 and 3). Several previous studies have indicated the roles of JA in aphid infestation responses, for example, $L O X$ genes were strongly up-regulated by Myzus persicae feeding on $A$. thaliana leaves [9], M. nicotianae feeding on Nicotiana attenuata leaves [30], and M. euphorbiae on tomato leaf tissues [31]. Infestation of potato (Solanum tuberosum L.) by $M$. persicae induced transcripts encoding $P R-1$, which increased gradually during the time-course of aphid feeding, and the expression of $J A Z 1$ was kept at a stable level [32]. In present study, three JAZ (Unigene11800_All, Unigene19974_All and Unigene28971_All) and five MYC2 genes (Unigene14746_All, Unigene19948_All, Unigene17336_All, Unigene28993_All and Unigene3689_All) were significantly differentially expressed in CK-VS-Y (Table 2). There were four differentially expressed $J A Z$ (Unigene11800_All, Unigene19974_All, Unigene28971_All and Unigene21174_All) and four MYC2 genes (Unige ne14746_All, Unigene19948_All, Unigene17336_All and Unigene28993_All) in CK-VS-Z (Table 3), indicating that Unigene3689_All might be related with the JA signaling pathway and play a major role in wound-induced response by aphid infestation. Jasmonate ZIM-domain proteins (JAZ) identified as key players of JA signaling cascade repress expression of JA-responsive genes by binding to transcriptional factors, such as MYC2 [33]. Plants increase the synthesis of JA which is then transformed to JA-Ile by jasmonic acid resistant 1 (JAR1) enzyme under stress [34]. The JA-Ile conjugate promotes interaction between JAZ and COI1 proteins in Skp/ Cullin/F-box complex $\left(\mathrm{SCF}^{\mathrm{COI1}}\right)$, resulting the degradation of JAZ through $\mathrm{SCF}^{\mathrm{COI1}}$-dependent $26 \mathrm{~S}$ proteasome pathway and the removal of inhibition to MYC2, thereby starting the transcription of JA-responsive genes, such as vegetative storage protein (VSP) gene [35-37]. How the JAZ and MYC2 regulate the response of chrysanthemum to aphid infestation is to be studied further. 
A

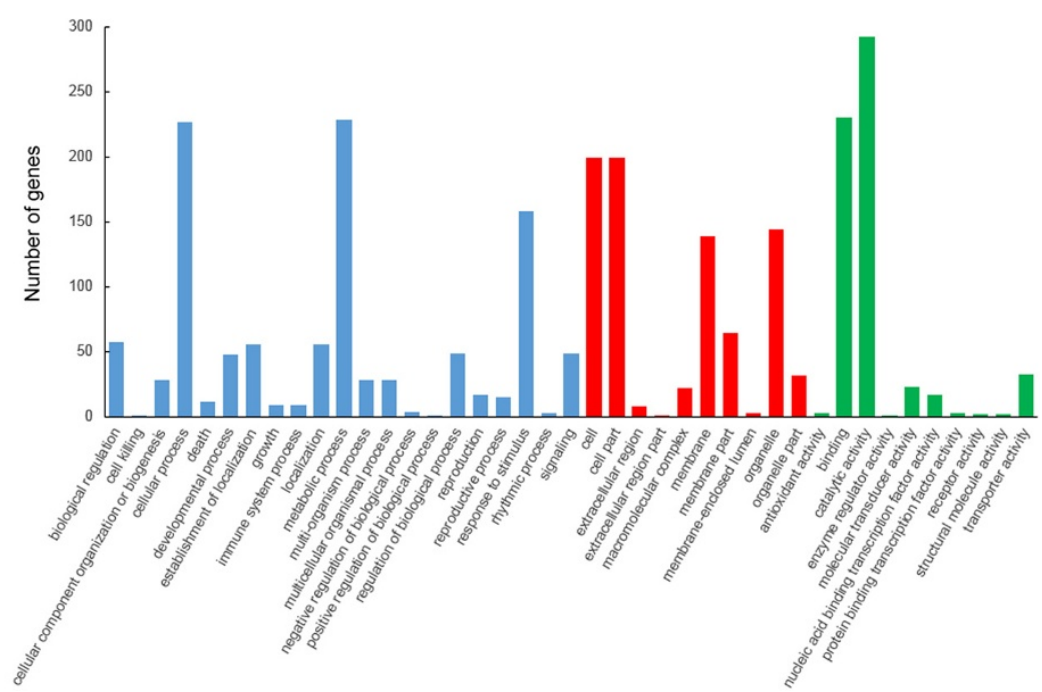

B

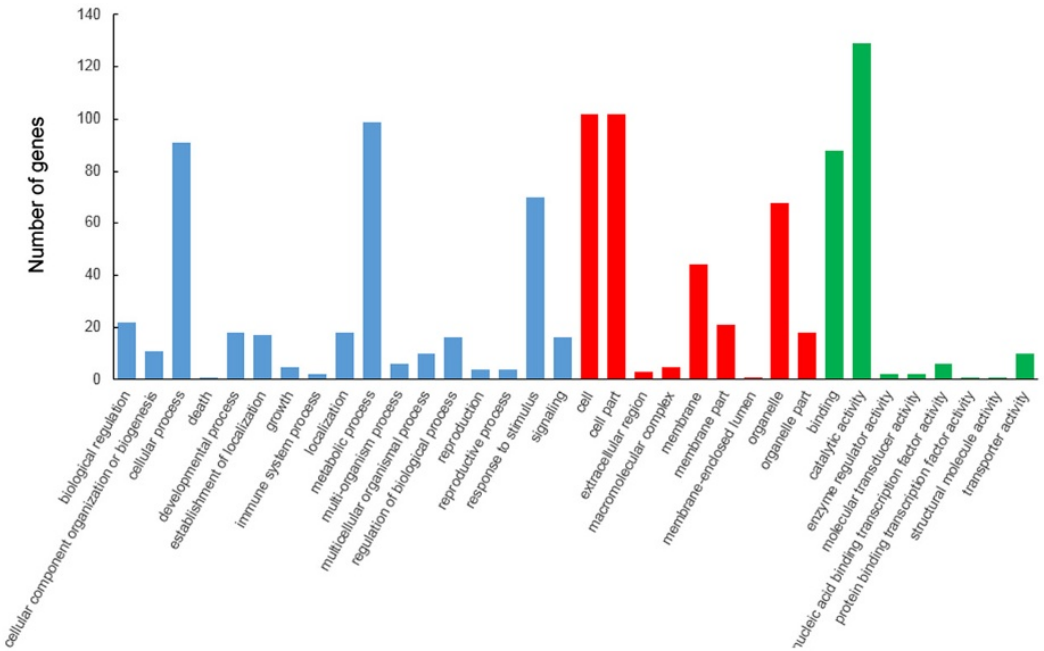

C

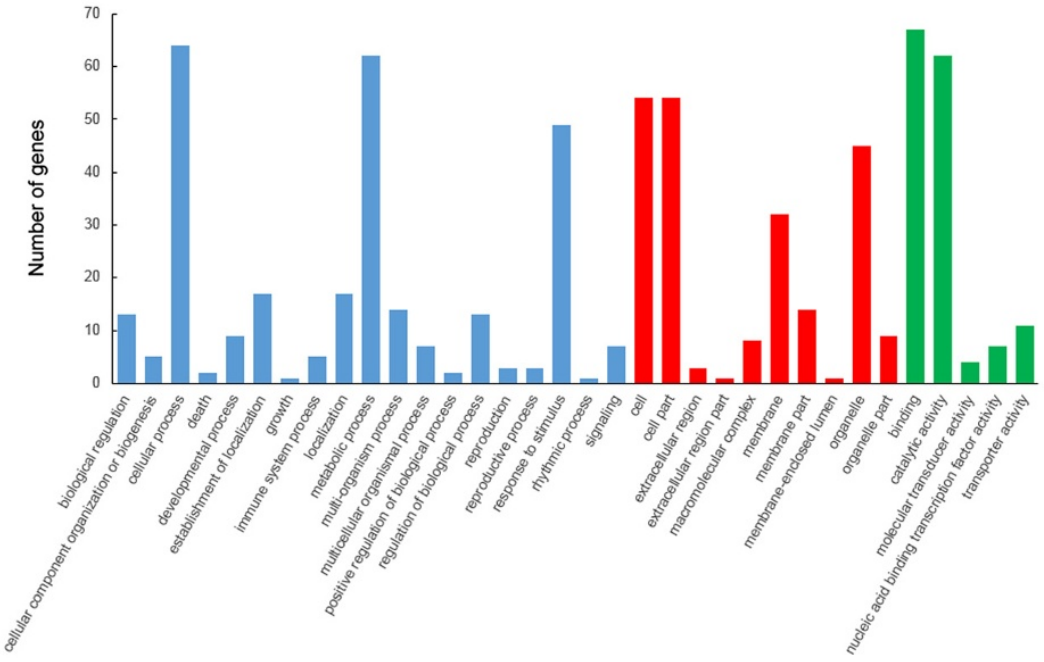

- Biological process

- Cellular component

- Molecular function

Figure 5 (See legend on next page.) 
(See figure on previous page.)

Figure 5 Gene Ontology (GO) functional classification of differentially expressed genes (DEGs). DEGs were annotated in three categories: biological process (blue), cellular component (red) and molecular function (green). CK: control; Y: aphid infestation treatment; Z: mock puncture treatment. A: comparison between library CK and Y (CK-VS-Y); B: comparison between library CK and Z (CK-VS-Z); C: comparison between library Z and Y (Z-VS-Y).

Though relatively few studies have been reported on the participation of ET in plant-aphid interactions, some studies have suggested that aphid infestation markedly increased the production of ET in leaves of plants, including barley [16], celery [38], Arabidopsis [19] and wheat [39]. Unigene10068_All, Unigene38824_All and Unigene1735_All encoding 1-aminocyclopropane1-carboxylic acid (ACC) synthases, the key enzymes in ET biosynthesis, were up-expressed in the CK-VS-Y (Table 2). ETHYLENE INSENSITIVE2 (EIN2), a membrane protein, plays an essential role in ET signaling pathway and is indispensable for defense responses. For instance, the EIN2 gene is demanded for the induced resistance to $M$. persicae in Arabidopsis treated by $\mathrm{HrpN}_{\mathrm{Ea}}$ [40]. Besides, ET signaling pathway through EIN2 results in transcription of the plant defensin gene PDF1.2, a molecular marker of ET

Table 2 Differentially expressed genes (DEGs) involved in phytohormone metabolism and signaling pathway in the comparison between CK and Y (CK-VS-Y)

\begin{tabular}{|c|c|c|c|c|c|c|c|}
\hline GeneID & CK-RPKM & Y-RPKM & $\begin{array}{c}\log _{2} \text { Ratio } \\
\text { (Y/CK) }\end{array}$ & $\begin{array}{l}\text { Up-Down-Regulation } \\
\text { (Y/CK) }\end{array}$ & P-value & FDR & Gene description \\
\hline Unigene107_All & 6.80 & 21.82 & 1.68 & up & $6.52 \mathrm{E}-15$ & $4.23 \mathrm{E}-13$ & NPR1-1 protein \\
\hline Unigene23699_All & 23.61 & 65.91 & 1.48 & up & $1.10 \mathrm{E}-21$ & $1.08 \mathrm{E}-19$ & NIM1-like protein 1 \\
\hline Unigene16290_All & 28.91 & 58.50 & 1.02 & up & $2.30 \mathrm{E}-05$ & 0.000575 & NIM1-like protein 1 \\
\hline Unigene2058_All & 4.07 & 13.85 & 1.77 & up & $4.50 \mathrm{E}-07$ & $1.47 \mathrm{E}-05$ & TGA transcription factor \\
\hline Unigene3706_All & 51.60 & 135.05 & 1.39 & up & $3.28 \mathrm{E}-68$ & $1.09 \mathrm{E}-65$ & TGA transcription factor \\
\hline Unigene11738_All & 5.11 & 21.60 & 2.08 & up & 2.73E-09 & $1.15 \mathrm{E}-07$ & Phospholipase A1 \\
\hline Unigene37023_All & 27.84 & 114.64 & 2.04 & up & $5.60 \mathrm{E}-20$ & $5.05 \mathrm{E}-18$ & Phospholipase A1 \\
\hline Unigene45678_All & 37.39 & 154.04 & 2.04 & up & $1.38 \mathrm{E}-107$ & 7.9E-105 & Lipoxygenase \\
\hline Unigene11030_All & 62.93 & 152.57 & 1.28 & up & $9.16 \mathrm{E}-44$ & $1.83 \mathrm{E}-41$ & Allene oxide cyclase \\
\hline Unigene29173_All & 85.71 & 199.13 & 1.22 & up & $6.06 \mathrm{E}-72$ & $2.09 \mathrm{E}-69$ & 12-oxophytodienoic acid reductase \\
\hline Unigene11800_All & 9.14 & 133.63 & 3.87 & up & 2.29E-105 & 1.25E-102 & $\begin{array}{l}\text { Jasmonate ZIM domain-containing } \\
\text { protein }\end{array}$ \\
\hline Unigene19974_All & 25.67 & 112.38 & 2.13 & up & $9.06 \mathrm{E}-53$ & $2.20 \mathrm{E}-50$ & $\begin{array}{l}\text { Jasmonate ZIM domain-containing } \\
\text { protein }\end{array}$ \\
\hline Unigene28971_All & 32.55 & 103.54 & 1.67 & up & $2.66 \mathrm{E}-35$ & $4.12 \mathrm{E}-33$ & $\begin{array}{c}\text { Jasmonate ZIM domain-containing } \\
\text { protein }\end{array}$ \\
\hline Unigene14746_All & 10.23 & 30.30 & 1.57 & up & $8.59 \mathrm{E}-10$ & $3.78 \mathrm{E}-08$ & MYC2 transcription factor \\
\hline Unigene19948_All & 25.16 & 67.21 & 1.42 & up & $2.18 \mathrm{E}-25$ & $2.48 \mathrm{E}-23$ & MYC2 transcription factor \\
\hline Unigene17336_All & 23.29 & 56.14 & 1.27 & up & $4.61 \mathrm{E}-23$ & $4.76 \mathrm{E}-21$ & MYC2 transcription factor \\
\hline Unigene28993_All & 38.28 & 88.92 & 1.22 & up & $2.33 \mathrm{E}-18$ & $1.89 \mathrm{E}-16$ & MYC2 transcription factor \\
\hline Unigene3689_All & 60.21 & 26.95 & -1.16 & down & $2.97 \mathrm{E}-26$ & $3.47 \mathrm{E}-24$ & MYC2 transcription factor \\
\hline Unigene10068_All & 55.68 & 122.23 & 1.13 & up & $8.46 \mathrm{E}-10$ & $3.73 \mathrm{E}-08$ & 1-aminocyclopropane-1-carboxylate synthase \\
\hline Unigene38824_All & 58.78 & 121.12 & 1.04 & up & 1.10E-08 & $4.32 \mathrm{E}-07$ & $\begin{array}{l}\text { 1-aminocyclopropane-1-carboxylate } \\
\text { synthase }\end{array}$ \\
\hline Unigene1735_All & 99.27 & 203.73 & 1.04 & up & $8.39 \mathrm{E}-15$ & $5.38 \mathrm{E}-13$ & $\begin{array}{l}\text { 1-aminocyclopropane-1-carboxylate } \\
\text { synthase }\end{array}$ \\
\hline Unigene23619_All & 12.85 & 57.09 & 2.15 & up & $2.21 \mathrm{E}-26$ & $2.59 \mathrm{E}-24$ & DELLA protein \\
\hline Unigene21755_All & 9.62 & 43.11 & 2.16 & up & $9.28 \mathrm{E}-12$ & $4.81 \mathrm{E}-10$ & DELLA protein \\
\hline Unigene29632_All & 99.04 & 300.15 & 1.60 & up & $6.89 \mathrm{E}-90$ & $3.08 \mathrm{E}-87$ & DELLA protein \\
\hline Unigene41060_All & 39.09 & 110.66 & 1.50 & up & $6.35 \mathrm{E}-12$ & $3.35 \mathrm{E}-10$ & DELLA protein \\
\hline Unigene21602_All & 24.78 & 54.70 & 1.14 & up & $1.41 \mathrm{E}-28$ & $1.81 \mathrm{E}-26$ & DELLA protein \\
\hline
\end{tabular}

The criteria used for assigning significance were: $P$-value $<0.05, \mathrm{FDR} \leq 0.001$, and absolute $\mid \log _{2}$ Ratio(Y/CK) $\mid \geq 1$. CK: control; Y: aphid infestation treatment. 
Table 3 Differentially expressed genes (DEGs) involved in phytohormone metabolism and signaling pathway in the comparison between CK and Z (CK-VS-Z)

\begin{tabular}{|c|c|c|c|c|c|c|c|}
\hline GeneID & CK-RPKM & Z-RPKM & $\begin{array}{c}\log _{2} \text { Ratio } \\
\text { (Z/CK) }\end{array}$ & $\begin{array}{l}\text { Up-Down-Regulation } \\
\text { (Z/CK) }\end{array}$ & P-value & FDR & Gene description \\
\hline Unigene107_All & 6.80 & 19.73 & 1.54 & up & $2.95 \mathrm{E}-12$ & $2.70 \mathrm{E}-10$ & NPR1-1 protein \\
\hline Unigene23699_All & 23.61 & 56.87 & 1.27 & up & $2.04 \mathrm{E}-15$ & $2.36 \mathrm{E}-13$ & NIM1-like protein 1 \\
\hline Unigene15228_All & 70.35 & 150.72 & 1.10 & up & $9.75 E-47$ & $3.75 \mathrm{E}-44$ & Phospholipase A1 \\
\hline Unigene37023_All & 27.84 & 65.81 & 1.24 & up & $1.20 \mathrm{E}-06$ & 5.99E-05 & Phospholipase A1 \\
\hline Unigene45678_All & 37.39 & 109.57 & 1.55 & up & $1.01 \mathrm{E}-53$ & 4.64E-51 & Lipoxygenase \\
\hline Unigene752_All & 1.22 & 8.31 & 2.77 & up & 1.65E-05 & 0.000669 & Lipoxygenase \\
\hline Unigene26067_All & 33.48 & 95.98 & 1.52 & up & $2.99 \mathrm{E}-72$ & $1.99 E-69$ & Allene oxide synthase \\
\hline Unigene11030_All & 62.93 & 216.68 & 1.78 & up & $1.32 \mathrm{E}-100$ & $1.49 \mathrm{E}-97$ & Allene oxide cyclase \\
\hline Unigene29173_All & 85.71 & 194.21 & 1.18 & up & $7.99 E-68$ & $4.81 \mathrm{E}-65$ & 12-oxophytodienoic acid reductase \\
\hline Unigene45901_All & 14.60 & 58.94 & 2.01 & up & $1.30 \mathrm{E}-30$ & $3.00 \mathrm{E}-28$ & 12-oxophytodienoic acid reductase \\
\hline Unigene11800_All & 9.14 & 169.72 & 4.21 & up & $3.46 \mathrm{E}-143$ & $6.12 \mathrm{E}-140$ & Jasmonate ZIM domain-containing protein \\
\hline Unigene19974_All & 25.67 & 146.79 & 2.52 & up & $3.21 \mathrm{E}-84$ & $2.52 \mathrm{E}-81$ & Jasmonate ZIM domain-containing protein \\
\hline Unigene28971_All & 32.55 & 105.47 & 1.70 & up & $4.12 \mathrm{E}-37$ & $1.16 \mathrm{E}-34$ & Jasmonate ZIM domain-containing protein \\
\hline Unigene21174_All & 36.91 & 120.43 & 1.71 & up & $2.79 \mathrm{E}-50$ & $1.22 \mathrm{E}-47$ & Jasmonate ZIM domain-containing protein \\
\hline Unigene14746_All & 10.23 & 51.02 & 2.32 & up & $1.74 \mathrm{E}-25$ & $3.29 \mathrm{E}-23$ & MYC2 transcription factor \\
\hline Unigene19948_All & 25.16 & 121.61 & 2.27 & up & $6.16 \mathrm{E}-85$ & $4.98 \mathrm{E}-82$ & MYC2 transcription factor \\
\hline Unigene17336_All & 23.29 & 60.28 & 1.37 & up & $6.73 E-28$ & $1.40 \mathrm{E}-25$ & MYC2 transcription factor \\
\hline Unigene28993_All & 38.28 & 106.40 & 1.47 & up & $6.39 E-29$ & $1.37 E-26$ & MYC2 transcription factor \\
\hline Unigene23619_All & 12.85 & 27.10 & 1.08 & up & $9.78 \mathrm{E}-06$ & 0.000418 & DELLA protein \\
\hline
\end{tabular}

The criteria used for assigning significance were: $P$-value $<0.05, F D R \leq 0.001$, and absolute $\mid \log _{2}$ Ratio(Z/CK) $\mid \geq 1$. CK: control; Z: mock puncture treatment.

signal transduction and needs EIN2 for transcription [40]. ET often works synergistically with JA [8]. Recent study shows that the ET-stabilized transcriptional factors (EIN3/EIL1) mediate several ET transcriptional responses that are regulated by crosstalk with JA, which enhances the activity of EIN3/EIL1 by removal of JAZ proteins repressing EIN3/EIL1 [41]. However, there is no EIN2, PDF1.2 or EIN3/EIL1 gene significantly differentially expressed in both CK-VS-Y and CK-VS-Z comparison, which may be related with the insensitivity of chrysanthemum to ethylene $[42,43]$, therefore, we suggested that the insensitivity of chrysanthemum to ET might partially compromise ET cascade or ET-mediated aphid infestation response in chrysanthemum in a different way from that in other plants.

Besides the SA-JA-ET backbone, other plant hormones, such as abscisic acid (ABA), auxin and gibberellin (GB), have gotten less attention as potential factors that mediate aphid resistance. However, these hormones also play a significant role in herbivore-induced defense responses, feeding into the SA-JA-ET network. ABA synthesis and cascades affect herbivore-activated JA metabolism and signaling in Arabidopsis [44], maize [45] and tomato [46]. Synergy between ABA and JA could stimulate MYC-dependent gene expression [47], and MYC2 functions as an integration point between the ABA and JA pathways
[48,49]. Auxin and JA co-regulate JAZ1 and MYC2 [50,51]. Interestingly, gibberellic acid (GA) affect the JA signaling pathway through competitively binding to JAZ proteins instead of DELLAs, negative regulators of GB signaling, thereby promoting MYC2-induced gene expression [52]. GA perception results in degradation of DELLAs, leading to the inhibition of MYC2 and attenuated JA responses. The expression of five DELLA genes (Unigene23619_All, Unigene21755_All, Unigene29632_All, Unigene41060_All and Unigene21602_All) was modulated by aphid infestation in CK-VS-Y (Table 2) and one gene (Unigene23619_All) by mock puncture experiment in CK and Z (Table 3), indicating the complex connections between different plant hormone signalings induced by aphid in chrysanthemum leaf.

\section{Transcription factors (TFs) responding to aphid infestation}

TFs are important regulators of plants' defense response. Several members of TF families have been reported to be involved in plant-herbivore interaction. Overexpression of OsWRKY89 increased the resistance of rice to whitebacked planthopper, Sogatella furcifera, a sap-sucking insect [53]. In Nicotiana attenuata, silencing WRKY3 and/or WRKY6 makes plants more susceptible to insect herbivory, and this susceptibility is connected with the impairment of 
Table 4 Differentially expressed WRKY, MYB, AP2/ERF, GRAS and HSF genes responding to aphid herbivory in the comparison between CK and Y (CK-VS-Y)

\begin{tabular}{|c|c|c|c|c|c|c|c|}
\hline GenelD & CK-RPKM & Y-RPKM & $\log _{2}$ Ratio(Y/CK) & Up-Down-Regulation(Y/CK) & P-value & FDR & Gene description \\
\hline Unigene12209_All & 20.74 & 92.22 & 2.15 & up & $3.73 \mathrm{E}-45$ & $7.69 \mathrm{E}-43$ & WRKY transcription factor \\
\hline Unigene41938_All & 43.08 & 183.74 & 2.09 & up & $9.59 \mathrm{E}-37$ & $1.56 \mathrm{E}-34$ & WRKY transcription factor \\
\hline Unigene32329_All & 10.38 & 43.97 & 2.08 & up & $2.25 \mathrm{E}-08$ & $8.60 \mathrm{E}-07$ & WRKY transcription factor \\
\hline Unigene10297_All & 85.74 & 348.25 & 2.02 & up & $3.24 \mathrm{E}-56$ & $8.40 \mathrm{E}-54$ & WRKY transcription factor \\
\hline Unigene37863_All & 85.47 & 340.39 & 1.99 & up & 7.04E-94 & $3.33 \mathrm{E}-91$ & WRKY transcription factor \\
\hline Unigene20571_All & 20.38 & 73.31 & 1.85 & up & $2.52 \mathrm{E}-19$ & $2.19 \mathrm{E}-17$ & WRKY transcription factor \\
\hline Unigene37259_All & 17.93 & 64.06 & 1.84 & up & $9.77 \mathrm{E}-11$ & 4.64E-09 & WRKY transcription factor \\
\hline Unigene37869_All & 268.18 & 945.12 & 1.82 & up & $1.82 \mathrm{E}-155$ & $2.00 \mathrm{E}-152$ & WRKY transcription factor \\
\hline Unigene7360_All & 74.03 & 251.85 & 1.77 & up & $6.60 \mathrm{E}-41$ & $1.20 \mathrm{E}-38$ & WRKY transcription factor \\
\hline Unigene37669_All & 337.88 & 1022.27 & 1.60 & up & $9.04 \mathrm{E}-90$ & $4.01 \mathrm{E}-87$ & WRKY transcription factor \\
\hline Unigene1677_All & 38.23 & 115.12 & 1.59 & up & $3.73 \mathrm{E}-18$ & $2.99 \mathrm{E}-16$ & WRKY transcription factor \\
\hline Unigene17473_All & 33.01 & 69.77 & 1.08 & up & $1.31 \mathrm{E}-07$ & 4.55E-06 & WRKY transcription factor \\
\hline Unigene6575_All & 23.05 & 118.37 & 2.36 & up & $3.80 \mathrm{E}-48$ & $8.14 \mathrm{E}-46$ & MYB transcription factor \\
\hline Unigene27371_All & 35.74 & 136.17 & 1.93 & up & $5.40 \mathrm{E}-40$ & $9.55 \mathrm{E}-38$ & MYB transcription factor \\
\hline Unigene29130_All & 35.63 & 95.08 & 1.42 & up & $1.66 \mathrm{E}-37$ & $2.74 \mathrm{E}-35$ & MYB transcription factor \\
\hline Unigene5110_All & 46.71 & 113.33 & 1.28 & up & $7.40 \mathrm{E}-29$ & $9.65 \mathrm{E}-27$ & MYB transcription factor \\
\hline Unigene20732_All & 27.31 & 61.70 & 1.18 & up & $2.12 \mathrm{E}-12$ & $1.18 \mathrm{E}-10$ & MYB transcription factor \\
\hline Unigene1509_All & 21.86 & 48.30 & 1.14 & up & 2.69E-07 & $9.03 \mathrm{E}-06$ & MYB transcription factor \\
\hline Unigene10992_All & 29.16 & 8.50 & -1.78 & down & $2.82 \mathrm{E}-16$ & $2.01 \mathrm{E}-14$ & MYB transcription factor \\
\hline Unigene33772_All & 33.67 & 115.91 & 1.78 & up & $1.32 \mathrm{E}-15$ & $9.07 \mathrm{E}-14$ & AP2/ERF transcription factor \\
\hline Unigene29332_All & 26.35 & 85.91 & 1.71 & up & 2.06E-18 & $1.68 \mathrm{E}-16$ & AP2/ERF transcription factor \\
\hline Unigene37496_All & 281.36 & 569.19 & 1.02 & up & $3.06 \mathrm{E}-30$ & $4.18 \mathrm{E}-28$ & AP2/ERF transcription factor \\
\hline Unigene20692_All & 107.36 & 250.56 & 1.22 & up & $6.69 \mathrm{E}-43$ & $1.30 \mathrm{E}-40$ & AP2/ERF transcription factor \\
\hline Unigene28929_All & 196.76 & 405.51 & 1.04 & up & $1.12 \mathrm{E}-67$ & $3.67 \mathrm{E}-65$ & AP2/ERF transcription factor \\
\hline Unigene20430_All & 11.14 & 71.20 & 2.68 & up & 4.27E-43 & $8.31 \mathrm{E}-41$ & AP2/ERF transcription factor \\
\hline Unigene21602_All & 24.78 & 54.70 & 1.14 & up & $1.41 \mathrm{E}-28$ & $1.81 \mathrm{E}-26$ & GRAS transcription factor \\
\hline Unigene23619_All & 12.85 & 57.10 & 2.15 & up & $2.21 \mathrm{E}-26$ & $2.59 \mathrm{E}-24$ & GRAS transcription factor \\
\hline Unigene11471_All & 9.76 & 31.50 & 1.69 & up & $5.41 \mathrm{E}-07$ & $1.74 \mathrm{E}-05$ & GRAS transcription factor \\
\hline Unigene41060_All & 39.09 & 111.00 & 1.50 & up & $6.35 \mathrm{E}-12$ & $3.35 \mathrm{E}-10$ & GRAS transcription factor \\
\hline Unigene24298_All & 32.99 & 76.36 & 1.21 & up & $6.17 \mathrm{E}-23$ & $6.34 \mathrm{E}-21$ & Heat shock factor \\
\hline Unigene3496_All & 27.02 & 104.20 & 1.95 & up & $2.08 \mathrm{E}-49$ & $4.55 \mathrm{E}-47$ & Heat shock factor \\
\hline Unigene24225_All & 14.71 & 5.05 & -1.54 & down & $1.58 \mathrm{E}-07$ & $5.46 \mathrm{E}-06$ & Heat shock factor \\
\hline
\end{tabular}

The criteria used for assigning significance were: $P$-value $<0.05, \mathrm{FDR} \leq 0.001$, and absolute $\mid \log _{2}$ Ratio(Y/CK) $\mid \geq 1$. CK: control; Y: aphid infestation treatment.

JA accumulation and defenses mediated by JA signaling [54], suggesting the crosstalk between TFs and phytohormone signaling. Silencing and overexpression of OSERF3 indicate that the gene is a central early herbivoreresponsive one that affects a set of defense-associated signaling pathways, such as MAPK cascades as well as SA, JA and ET signaling, and it acts as a vital switch modulating defense responses against chewing and piercing/sucking insects in rice [55]. The aphid-susceptible atmyb44 mutant showed a much greater susceptibility to aphid feeding and most compromised in induced resistance in Arabidopsis. A further stud shows that atmyb44 incurred an abolishment of the induction of EIN2, indicating a close link between AtMYB44 and EIN2 [40]. Besides their direct functions in plant-aphid interaction, TFs may also regulate the growth and development of plants to mediate defense responses indirectly, including photosynthesis, cell wall formation, carbon metabolism and so on. In present study, we also identified several differentially expressed TFs that were reported previously, including WRKY, MYB and AP2/ERF, and some new TFs responding to aphid herbivory in chrysanthemum, such as GRAS and HSF genes (Tables 4 and 5). In the CK-VS-Y, twelve WRKY, seven $M Y B$, six $A P 2 / E R F$, four GRAS and three HSF genes were recognized, 
Table 5 Differentially expressed WRKY, MYB, AP2/ERF, GRAS and HSF genes responding to aphid herbivory in the comparison between CK and Z (CK-VS-Z)

\begin{tabular}{cccccccc}
\hline GenelD & CK-RPKM & Z-RPKM & log $_{\mathbf{2}}$ Ratio(Z/CK) & Up-Down-Regulation(Z/CK) & P-value & FDR & Gene description \\
\hline Unigene26514_All & 21.32 & 50.79 & 1.25 & up & $4.13 \mathrm{E}-06$ & 0.000192 & WRKY transcription factor \\
Unigene20571_All & 20.38 & 46.36 & 1.19 & up & $2.41 \mathrm{E}-07$ & $1.31 \mathrm{E}-05$ & WRKY transcription factor \\
Unigene29130_All & 35.63 & 82.07 & 1.20 & up & $5.25 \mathrm{E}-26$ & $1.02 \mathrm{E}-23$ & MYB transcription factor \\
Unigene33100_All & 10.52 & 43.44 & 2.05 & up & $6.04 \mathrm{E}-19$ & $8.69 \mathrm{E}-17$ & MYB transcription factor \\
Unigene33772_All & 33.67 & 88.24 & 1.39 & up & $5.09 \mathrm{E}-09$ & $3.43 \mathrm{E}-07$ & AP2/ERF transcription factor \\
Unigene29332_All & 26.35 & 59.86 & 1.18 & up & $2.45 \mathrm{E}-08$ & $1.52 \mathrm{E}-06$ & AP2/ERF transcription factor \\
Unigene20430_All & 11.14 & 66.16 & 2.57 & up & $7.72 \mathrm{E}-39$ & $2.25 \mathrm{E}-36$ & AP2/ERF transcription factor \\
Unigene23619_All & 12.85 & 27.10 & 1.08 & up & $9.78 \mathrm{E}-06$ & 0.000418 & GRAS transcription factor \\
Unigene3496_All & 27.02 & 62.69 & 1.21 & up & $3.72 \mathrm{E}-16$ & $4.59 \mathrm{E}-14$ & Heat shock factor \\
\hline
\end{tabular}

The criteria used for assigning significance were: $P$-value $<0.05, F D R \leq 0.001$, and absolute $\mid \log _{2}$ Ratio(Z/CK) $\mid \geq 1$. CK: control; Z: mock puncture treatment.

whereas only two WRKY genes, two $M Y B$ genes, three $A P 2 / E R F$ genes, one GRAS genes and one HSF genes were identified in the CK-VS-Z, implying that aphid feeding has bigger influences on gene expression and is more complicated than mock puncture treatment, and the new discovered aphid-responsive TFs, GRAS (Unigene21602_All, Unigene11471_All and Unigene41060_All) and HSF genes (Unigene24298_All and Unigene24225_All), might express specifically to aphid infestation. Still, the potential roles of these TFs need further investigation.

\section{Reactive oxygen species (ROS) and antioxidant genes}

Besides being toxic byproducts of metabolism, ROS, for example hydrogen peroxide $\left(\mathrm{H}_{2} \mathrm{O}_{2}\right)$, are also involved in the complex signaling network of plants $[56,57]$. There are at least three possible roles for ROS in plant-aphid interaction: direct adverse influences on aphid midgut tissues [8], triggering programmed cell death (PCD) [58] leading to apoptosis to against biotrophic aphids and stimulating defense signaling pathways towards aphid attack [56]. Aphid feeding alters plant redox state and induces the production of ROS [8], and others could also elicit the accumulation of ROS content, such as SA and JA, indicating possible interactions between ROS signaling and phytohormone transduction. Research of Russian wheat aphid [59], Diuraphis noxia (Mordvilko) infestation on wheat (Triticum aestivum L.) resulted in induction of $\mathrm{H}_{2} \mathrm{O}_{2}$ content and activity of NADPH oxidase from which ROS are largely derived [60], and strongly indicated a probable signaling role for $\mathrm{H}_{2} \mathrm{O}_{2}$. Here, three NADPH oxidase genes, Unigene45792_All, Unigene300_All and Unigene3581_All, were recognized in CK and Y comparison alone (Additional file 8: Table S7). Furthermore, enzymes, such as peroxidase (POD), ascorbate peroxidase (APX) and polyphenol oxidase (PPO), involved in ROS scavenging were also up-regulated during aphid infestation (Additional file 8: Table S7 and Additional file 9: Table S8), suggesting the maintenance of redox homeostasis is important for responses to aphid, which are consistent with our previous observation of the enhanced enzyme activities by aphid

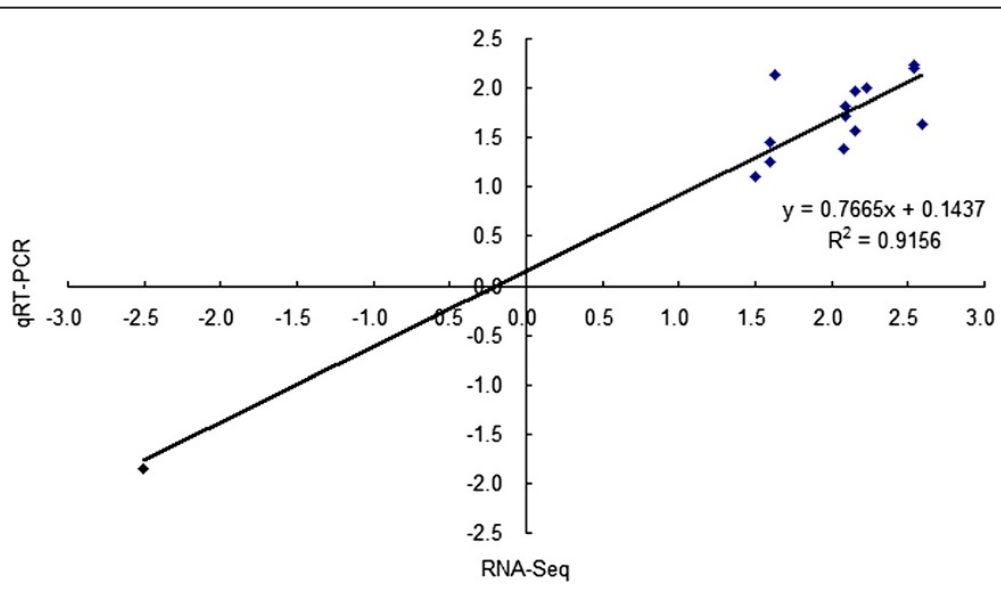

Figure 6 Quantitative real-time PCR (qRT-PCR) validation of differentially expressed genes (DEGs) from RNA-Seq in leaf tissues of chrysanthemum. Correlation of fold change analyzed by RNA-Seq platform ( $x$ axis) with data obtained using qRT-PCR (y axis). 
infestation. Except linked with detoxification of ROS, enzymes, such as peroxidases, are yet prerequisites for plant cell wall building [61], further demonstrating the complex regulatory network inside plants.

\section{Photosynthesis-associated genes involved in response to aphid feeding}

Aphids, phloem-feeding herbivores, drain plant nutrients of which the main components are saccharides resulting from photosynthesis. Saccharides drained from the sieve element are easy to be contaminated by bacteria on the surface of leaves, thereby affecting photosynthesis. In our study, only two photosynthesis-related genes (Unigene24131_All and Unigene9460_All) were detected in the $\mathrm{CK}$ and $\mathrm{Y}$ alone (Additional file 14: Table S13), both of them belonging to the components of photosystem were induced by aphids, which may suggest the strengthening of photosynthesis, compensating for the loss of nutrients and maintaining the normal growth processes. $D$. noxia feeding on leaves of wheat [39], M. persicae feeding on celery foliage [38] and $M$. nicotianae feeding on $N$. attenuata leaves tissues [30] promote the expression of photosynthesis genes, while some of them are decreased by $M$. nicotianae [30] or Schizaphis graminum [62], possibly reflecting the redistribution of metabolites from normal growth functions to defensive roles after aphids feeding in plants.

\section{Nucleotide-binding site-leucine-rich repeat (NBS-LRR) genes}

Two cloned aphid resistance $(R)$ genes, $M i-1.2$, conferring resistance to the potato aphid, Macrosiphum euphorbiae (Thomas) [3,4], and Vat, mediating resistance to the cotton aphid, Aphis gossypii Glover [5,63], belong to NBS-LRR family. Similarly, other plant-aphid interactions have revealed a tight relationship between NBS-LRR genes and resistance loci. Plants of wheat having $D$. noxia resistance gene contain leucine zipper (LZ)-NBS-LRR sequences [64-66]. Swanepoel and co-workers [67] also discovered tight connection between LZ-NBS-LRR sequence and $D$. noxia resistance gene. On the chromosome of Medicago truncatula, a locus which controls the resistance to the blue alfalfa aphid, Acyrthosiphon kondoi, is flanked by coiled-coil (CC)-NBS-LRR sequence [7]. Similarly, we found two differentially expressed genes (Unigene3633_All and Unigene14351_All) containing NBS-LRR region in CK-VS-Y (Additional file 15: Table S14). Further cloning and functional identification regarding the two genes would be necessary.

\section{Genes involved in cell wall biosynthesis}

In Arabidopsis, several COBRA and COBRA-like genes have been identified to be important for secondary cell wall development [68]. Loss of function mutation of these genes results in brittle stalks and decreased cellulose content [69], indicating that these genes are essential for normal cellulose deposition in secondary cell wall. Mutations in brittle culm1 (bc1) which encodes a COBRA-like protein suggest that it controls the mechanical strength of monocots and is an important player in the biosynthesis of cell walls of mechanical tissues [70]. There are three COBRA-like genes (Unigene11326_All, Unigene2724_All and Unigene22759_All) identified in CKVS-Y, and two (Unigene11326_All and Unigene2724_All) out of three in CK-VS-Z (Additional file 10: Table S9 and Additional file 11: Table S10). Hemicelluloses and pectins, which are both synthesized in the Golgi, and cellulose and callose, both synthesized at plasma membrane, are the major polysaccharides of the plant cell wall. The identification of cellulose synthase A (CesA), which is the catalytic subunit of the cellulose synthase complex [71,72], greatly enriches our understanding of the biosynthesis of cell wall polysaccharides. And some cellulose synthase-like (Csl) genes have also been reported to be responsible for the biosynthesis of glycan backbones in the Golgi [73]. In this study, two (Unigene25922_All and Unigene6200_All) and three Csl genes (Unigene3108_All, Unigene25922_All and Unigene6200_All) were detected in the CK-VS-Y and CK-VS-Z, respectively (Additional file 10: Table S9 and Additional file 11: Table S10). The up-regulation of COBRA-like and Csl genes suggests that the mechanical strength of the plant are somewhat strengthened, which might therefore hinder the puncturing of the aphid stylet during aphid feeding. Therefore, the detailed mechanisms of these genes during plant-aphid interactions could be another interesting topic, and relevant transgenic work would be more practical.

\section{Secondary metabolites}

Secondary metabolites, such as flavonoids, terpenes, phenolics and alkaloids, having antixenotic or antibiotic properties, could function in plant defense against herbivores [74]. In Vigna [75], there is a positive relationship between resistance or susceptibility properties against aphids and flavonoid glycoside content. The content of flavonoid in susceptible lines was lower than in resistant ones. In vitro bioassays proved that quercetin and isorhamnetin, members of endogenous flavonoids, have a significant inhibitory on the reproduction rate of aphids. In contrast, overexpression of AtMYB75, resulting in increasing flavonol levels, did enhance the resistance to caterpillars, but with no effects on the performance of B. brassicae [76]. Flavonoids, including flavones and isoflavones [77], are derived from the phenylpropanoid pathway, which is catalyzed by a number of enzymes, for example, PAL (phenylalanine ammonia-lyase), which is well studied for plant responses to biotic and abiotic stress. In this study, we got several DEGs related with flavonoids synthesis, such as PAL, in both CK-VS-Y and CK-VS-Z 
(Additional file 12: Table S11 and Additional file 13: Table S12). Attacked by herbivores, some plants would emit volatile compounds, which are mainly mono- and sesquiterpenes, used by parasitic wasps to find their hosts, the lepidopteran larvae. Terpene synthases catalyze the committed step in the biosynthesis of varieties of mono- and sesquiterpene products from prenyl diphosphate precursors. The expression of terpene synthase 1 (tps1) in the maize cv B73 was stimulated by herbivory and mechanical damage. Further analysis shows that the transcription of tps 1 or its homolog varies between different cultivars of maize [78]. Our previous study found that the increased content of monoterpenoids and sesquiterpenoids in the leaves of the hybrid between chrysanthemum and Artemisia vulgaris enhanced plant resistance to aphid [79]. Interestingly, two terpene synthase encoding genes (Unigene3919_All and Unigene26695_All) were detected in CK-VS-Y and CK-VS-Z, respectively (Additional file 12: Table S11 and Additional file 13: Table S12). These discussed above illustrate the involvement of secondary metabolites during aphid herbivory in chrysanthemum leaf, indicating their potential roles in the defense responses against aphids.

\section{Aphid feeding and mock puncture treatment}

Here, in our research, we conducted a mock puncture treatment trying to partially simulate the mechanical stress resulting from aphid penetration. Despite there are some differences between aphid stylet and puncture. For instance, aphid stylets were often wrapped by saliva which contains a complex mixture of enzymes and can induce defense responses [80]. Also, the mechanical degree of puncture treatment should be different from aphid stylets. Results that were discussed above show that it does have some similarities between aphid feeding and puncture treatment, such as genes involved in phytohormone metabolism and signaling pathway, ROS scavenging and cell wall biosynthesis, and some genes specifically expressed in response to aphid treatment, for example, NBS-LRR genes. And as shown in Figure 4B, 648 DEGs were specifically expressed in CK-VS-Y; 328 DEGs were co-expressed in CK-VS-Y and CK-VS-Z, suggesting that genes co-expressed in response to aphid feeding and puncture treatment might be involved in wound-induced response by aphid, otherwise genes may specifically respond to aphid sucking. These will allow us to figure out the potential impacts of aphid stylets and refine the processes of defense responses.

\section{Conclusions}

Taken together, these examples indicate that aphid feeding does have a global effect on gene expression in chrysanthemum leaf, including genes involved in phytohormone signaling, cell wall biosynthesis, photosynthesis, reactive oxygen species (ROS) pathway and transcription factors (TF), and so on. Usually, there are cross-communications between different defense pathways those genes belonging to, which provide an ability that allows plants to integrate environmental, developmental and defense-related signals, fine-tuning its defense responses.

\section{Methods}

\section{Plants growth}

Chrysanthemum morifolium 'nannongxunzhang' (aphid resistant) was obtained from the Chrysanthemum Germplasm Resource Preserving Centre, Nanjing Agricultural University, China. Seedlings were grown in $12 \mathrm{~cm}$ pots with a 1:2 mixture of vermiculite and garden soil without fertilizer. Plants were grown under a $16 \mathrm{~h}$ photoperiod $\left(160 \mu \mathrm{mol} \mathrm{m}^{-2} \mathrm{~s}^{-1}\right.$ photon flux density), a relative humidity of $80 \%$, and a day/night temperature of $25 / 18^{\circ} \mathrm{C}$ in a greenhouse. Uniformity plants grown to the 6-8 leaf stage were selected for further experiment.

\section{Aphid infestation and mock puncture treatment}

Aphids (Macrosiphoniella sanbourni Gillette) were collected from field-grown chrysanthemum plants, two instars nymphs were fostered and chosen to inoculate plants. For aphid infestation treatment $(Y)$, the third fully expanded leaves from stem tip were infested with twenty second instar aphids transferred by a soft brush. The infested leaves were caged with transparent ventilated plastic cages $(2 \mathrm{~cm}$ height $\times 5 \mathrm{~cm}$ diameter) sealed at the base of the petiole, equal to the leaves of control $(\mathrm{CK})$ and mock puncture treatment $(\mathrm{Z})$. For the mock puncture treatment $(Z)$, designed to partially simulate the mechanical stress resulting from aphid penetration, the third fully expanded leaf of each plant was punctured 5 times at $0 \mathrm{~h}, 10$ times at $24 \mathrm{~h}$, and 15 times at $48 \mathrm{~h}$ with a needle (approximately $0.30 \mathrm{~mm}$ diameter) [9]. Leaves of three seedlings for each treatment were harvested at $0 \mathrm{~h}, 3 \mathrm{~h}, 6 \mathrm{~h}, 12 \mathrm{~h}$, $24 \mathrm{~h}, 48 \mathrm{~h}$. Before harvest, aphids were removed by spraying with $1 \%(\mathrm{v} / \mathrm{v})$ SDS solution, which caused aphids to remove their mouthparts from plant tissues and then removed the aphids from the leaves by flushing the plants with deionized water. Harvested materials were immediately frozen in liquid nitrogen and stored at $-80^{\circ} \mathrm{C}$ for the following experiments. The samples collected at defined time points of each treatment were pooled for RNA-Seq.

\section{RNA extraction, cDNA library construction and Illumina sequencing}

Total RNA from leaf tissue of three separate libraries (CK, Z, Y) was extracted using RNAiso reagent (TaKaRa, Japan), following the manufacturer's instructions. The 
integrity and quality of the total RNA was evaluated using a 2100 Bioanalyzer RNA Nano chip device (Agilent, Santa Clara, CA, USA) and agarose gel electrophoresis, and the concentration was measured with a ND -1000 spectrophotometer (NanoDrop, Wilmington, DE).

The mRNA of each library was enriched using poly(T) oligonucleotide-attached magnetic beads. Following purification, the mRNA was fragmented to a size of $\sim 200 \mathrm{bp}$, and the RNA fragments were copied into first-strand cDNA using random hexamer-primed reverse transcription. Second-strand cDNA synthesis was generated using RNaseH and DNA polymerase I, and the cDNA fragments were processed for end repair, an addition of a single "A" base, and ligation of the adapters following Illumina's protocols and sequenced on Illumina $\mathrm{HiSeq}^{\mathrm{TM}} 2000$ platform.

\section{Processing of sequence data}

The raw reads from Illumina sequencing were initially processed to remove adaptor sequences and low-quality reads. The remaining reads called clean reads were then mapped to the set of chrysanthemum unigene sequences using SOAPaligner/SOAP2. No more than two mismatches were allowed for alignment. RPKM (reads per kb per million reads) was used to describe the expression levels of genes. Differential expression of the three libraries was based on the $\log _{2}$ ratio of the RPKM values. FDR (false discovery rate) providing a criterion to determine the $P$-value threshold in multiple tests and analyses was also applied to identify differential expressed genes. A stringent cutoff, the $P$-value $<0.05$, the FDR $\leq 0.001$ and $\mid \log _{2}$ Ratio $\mid \geq 1.0$, was used for determining differential expressed genes. Gene ontology (GO) was used to describe the function of these genes, and a hypergeometric test was used to map them to GO terms based on the BGI WEGO (Web Gene Ontology Annotation Plot, http://wego.genomics.org.cn/cgi-bin/wego/index.pl). All sequencing data have been deposited at the sequence read archive (SRA) of NCBI.

\section{Quantitative real-time PCR (qRT-PCR) validation}

qRT-PCR was carried out using a Eppendorf AG 22331 Hamburg thermocycler. The samples collected at different time points were pooled. Three independent biological replicates of each sample and three technical replicates of each biological replicate were used for qRT-PCR analysis. For each sample, 1 ug of total RNA removed DNA by RNase-free DNase I treatment was converted into cDNA using a Super RT kit (BioTeke, Beijing, China). And qRT-PCR was performed in a 20 ul volume containing $10 \mathrm{ul}$ SYBR Green PCR master mix (TaKaRa, Japan), $0.2 \mathrm{uM}$ of each primer (Table 6) and $10 \mathrm{ng}$ cDNA, and the amplification programme including an initial denaturation at $95^{\circ} \mathrm{C}$ for $60 \mathrm{~s}$, followed by 40 cycles of $95^{\circ} \mathrm{C}$ for $15 \mathrm{~s}, 55^{\circ} \mathrm{C}$ for $15 \mathrm{~s}$ and $72^{\circ} \mathrm{C}$ for $20 \mathrm{~s}$ ). At the end of the cycling process, a melting-curve analysis from 55 to $95^{\circ} \mathrm{C}$ with a heating rate of $0.5^{\circ} \mathrm{C} \mathrm{s}^{-1}$ was performed to determine specificity of amplified products. The chrysanthemum EF1 $\alpha$ gene was used as a reference. Relative expression levels were calculated using the ${ }_{2-}{ }^{\Delta \Delta C T}$ method.

\section{Availability of supporting data}

The data sets supporting the results of this article are available in the NCBI Sequence Read Archive (SRA) database under accession number SRP042216, http://www.ncbi. nlm.nih.gov/sra/?term=SRP042216.

Table 6 Primers of quantitative real-time PCR (qRT-PCR) validation of differentially expressed genes (DEGs)

\begin{tabular}{|c|c|c|c|}
\hline Gene ID & Forward primer & Reverse primer & Annotation \\
\hline Unigene12209_All & GTGGCTGAGATTGGTGGTTT & GCCTIACAAGCGTTCAGC & WRKY family transcription factor \\
\hline Unigene14378_All & TTAAGTCGGTITTCGGCTTG & GCATCCTCTTCGATCCTTTG & WRKY family transcription factor \\
\hline Unigene14705_All & GACCGTCAAGAACAGGGGTA & ATAGAAGGTCCCGCAAACCT & Protein kinase \\
\hline Unigene23047_All & GCCACAAAATCCGTCAACTT & GCCTAACGATCCCTTGTGAA & SAUR family gene \\
\hline Unigene22169_All & GTCAAATGCTGCAAGGGATT & ATCAACACTTGCCCGAAGAC & Disease resistance protein \\
\hline Unigene22508_All & CGCGTTCTTTCATTCCATT & CGGTCGAACCCAGATTTAAG & Kinase \\
\hline Unigene32329_All & TCGTACCGCTGGGAATITAG & TGGGCTCGACTCGACTACTT & WRKY family transcription factor \\
\hline Unigene23619_All & ATGGGTGTTACAGGGATGGA & ACACAGGAGAGCTCCAGGAA & GRAS family transcription factor \\
\hline Unigene29632_All & CCTCCTAAGCTTCGCATCAC & GCTGTTAACCGCTGACCAAT & gibberellin-responsive protein \\
\hline Unigene41060_All & GTAATCTGGAGCATGGGTGG & CTIAATGGTGTGCCCGTTC & GRAS family transcription factor \\
\hline Unigene36228_All & GGTTGTTGGTTCTCGGAAA & TACCAACAGTAACACCGCCA & Protein kinase \\
\hline Unigene41938_All & GAGGATITCGCTGCCTIA & TCAACCACAAGAATGGAGCA & WRKY family transcription factor \\
\hline Unigene49088_All & ACACTIGTITCGGTTGGG & GCAAGACCAACCATGAGGAT & Protein kinase \\
\hline Unigene55750_All & ACCAGGATAAGGGAAGACGG & TCCATCCCAAATTTCCAAAA & protein with unknown function \\
\hline
\end{tabular}




\section{Additional files}

Additional file 1: Figure S1. Component of the raw reads in the three RNA libraries. "Clean reads" are those remaining after removal of adaptor sequences and low-quality reads. The numbers in parentheses indicate the percentage of each type of read present. CK: control; Y: aphid infestation treatment; Z: mock puncture treatment.

Additional file 2: Table S1. Differentially expressed genes (DEGs) in the comparison between libraries CK and Y. CK: control; Y: aphid infestation treatment. The criteria used for assigning significance were: $P$-value $<0.05$, FDR $\leq 0.001$, and estimated absolute $\left|\log _{2} \operatorname{Ratio}(Y / C K)\right| \geq 1$. Genes listed in descending order of absolute $\mid \log _{2}$ Ratio(Y/CK)|. GenelDs got from the Chrysanthemum Reference Sequence Database. Annotation of unigene sequences performed using BlastX $(E<10)$. The "GeneLength" column gives the length of exon sequence. CK- and Y-expression: frequency of unigene transcripts in libraries CK and Y, respectively. CK- and Y-RPKM: reads per kb per million reads for each unigene in libraries $C K$ and $Y$, respectively. $\mathrm{Log}_{2}$ Ratio(Y/CK): the ratio between the RPKM in Y and the RPKM in CK. KEGG: annotation according to the KEGG database by BLAST. Blast nr: identification of homologues in GenBank. GO Component, GO Function and Go Process: ontology information of Cellular Components, Molecular Function and Biological Processes of Gene-corresponding GO terms. "-": no hit.

Additional file 3: Table S2. Differentially expressed genes (DEGs) in the comparison between libraries CK and Z. CK: control; Z: mock puncture treatment. The criteria used for assigning significance were: $P$-value $<0.05$, FDR $\leq 0.001$, and estimated absolute $\left|\log _{2} R a t i o(Z / C K)\right| \geq 1$. Genes listed in descending order of absolute $\| \log _{2} R a t i o(Z / C K) \mid$. GenelDs got from the Chrysanthemum Reference Sequence Database. Annotation of unigene sequences performed using BlastX ( $E<10)$. The "GeneLength" column gives the length of exon sequence. CK- and Z-expression: frequency of unigene transcripts in libraries CK and Z, respectively. CK- and Z-RPKM: reads per $\mathrm{kb}$ per million reads for each unigene in libraries $C K$ and $Z$, respectively. $\log _{2}$ Ratio(Z/CK): the ratio between the RPKM in Z and the RPKM in CK. KEGG: annotation according to the KEGG database by BLAST. Blast nr: identification of homologues in GenBank. GO Component, GO Function and Go Process: ontology information of Cellular Components, Molecular Function and Biological Processes of Gene-corresponding GO terms. "-": no hit.

Additional file 4: Table S3. Differentially expressed genes (DEGs) in the comparison between libraries $Z$ and $Y$. Z: mock puncture treatment; $Y$ : aphid infestation treatment. The criteria used for assigning significance were: $P$-value $<0.05, F D R \leq 0.001$, and estimated absolute $\| \log _{2}$ Ratio $(Y / Z) \mid \geq 1$. Genes listed in descending order of absolute |log 2 Ratio(Y/Z)|. GenelDs got from the Chrysanthemum Reference Sequence Database. Annotation of unigene sequences performed using BlastX $(E<10)$. The "GeneLength" column gives the length of exon sequence. $Z$ - and $Y$-expression: frequency of unigene transcripts in libraries $Z$ and $Y$, respectively. Z- and Y-RPKM: reads per kb per million reads for each unigene in libraries $Z$ and $Y$, respectively. $\log _{2}$ Ratio(Y/Z): the ratio between the RPKM in Y and the RPKM in Z. KEGG: annotation according to the KEGG database by BLAST. Blast nr: identification of homologues in GenBank. GO Component, GO Function and Go Process: ontology information of Cellular Components, Molecular Function and Biological Processes of Gene-corresponding GO terms. "-": no hit.

Additional file 5: Table S4. GO classification of differentially expressed genes (DEGs) in the comparison between library CK and Y. CK: control; Y: aphid infestation treatment.

Additional file 6: Table S5. GO classification of differentially expressed genes (DEGs) in the comparison between library CK and Z. CK: control; Z: mock puncture treatment.

Additional file 7: Table S6. GO classification of differentially expressed genes (DEGs) in the comparison between library $Z$ and $Y$. Z: mock puncture treatment; $Y$ : aphid infestation treatment.

Additional file 8: Table S7. Differentially expressed NADPH oxidase genes and enzymes involved in reactive oxygen species (ROS) scavenging responding to aphid herbivory in the comparison between CK and $Y(C K-V S-Y)$. The criteria used for assigning significance were: $P$-value $<0.05, F D R \leq 0.001$, and $\mid \log _{2}$ Ratio(Y/CK) $\mid \geq 1$. RPKM: reads per kb per million reads. CK: control; Y: aphid infestation treatment.
Additional file 9: Table S8. Enzymes involved in reactive oxygen species (ROS) scavenging responding to aphid herbivory in the comparison between CK and Z (CK-VS-Z). The criteria used for assigning significance were: $P$-value $<0.05$, FDR $\leq 0.001$, and $\mid \log _{2}$ Ratio(Z/CK) $\mid \geq 1$. RPKM reads per kb per million reads. CK: control; Z: mock puncture treatment.

Additional file 10: Table S9. Differentially expressed genes (DEGs) involved in cell wall biosynthesis responding to aphid herbivory in the comparison between CK and $Y$ (CK-VS-Y). The criteria used for assigning significance were: $P$-value $<0.05, \mathrm{FDR} \leq 0.001$, and $\mid \log _{2}$ Ratio(Y/CK) $\mid \geq 1$. RPKM: reads per kb per million reads. CK: control; Y: aphid infestation treatment.

Additional file 11: Table S10. Differentially expressed genes (DEGs) involved in cell wall biosynthesis responding to aphid herbivory in the comparison between CK and Z (CK-VS-Z). The criteria used for assigning significance were: $P$-value $<0.05, F D R \leq 0.001$, and $\mid \log _{2}$ Ratio(Z/CK) $\mid \geq 1$. RPKM: reads per kb per million reads. CK: control; Z: mock puncture treatment.

Additional file 12: Table S11. Differentially expressed genes (DEGs) involved in secondary metabolites responding to aphid herbivory in the comparison between CK and $Y$ (CK-VS-Y). The criteria used for assigning significance were: $P$-value $<0.05$, FDR $\leq 0.001$, and $\mid \log _{2}$ Ratio $(Y / C K) \mid \geq 1$. RPKM: reads per kb per million reads. CK: control; Y: aphid infestation treatment.

Additional file 13: Table S12. Differentially expressed genes (DEGs) involved in secondary metabolites responding to aphid herbivory in the comparison between CK and Z (CK-VS-Z). The criteria used for assigning significance were: $P$-value $<0.05$, FDR $\leq 0.001$, and $\mid \log _{2}$ Ratio(Z/CK) $\mid \geq 1$. RPKM: reads per kb per million reads. CK: control; Z: mock puncture treatment.

Additional file 14: Table S13. Differentially expressed photosynthesis-related genes responding to aphid herbivory in the comparison between CK and $Y$ (CK-VS-Y). The criteria used for assigning significance were: $P$-value $<0.05, \mathrm{FDR} \leq 0.001$, and $\mid \log _{2}$ Ratio(Y/CK) $\mid \geq 1$. RPKM: reads per kb per million reads. CK: control; Y: aphid infestation treatment.

Additional file 15: Table S14. Differentially expressed nucleotide-binding site-leucine-rich repeat (NBS-LRR) genes responding to aphid herbivory in the comparison between CK and Y (CK-VS-Y). The criteria used for assigning significance were: $P$-value $<0.05, F D R \leq 0.001$, and $\left|\log _{2} R a t i o(Y / C K)\right| \geq 1$. RPKM: reads per kb per million reads. CK: control; $Y$ : aphid infestation treatment.

\section{Competing interests}

The authors declare that they have no competing interests.

\section{Authors' contributions}

XX and YS performed the research and wrote the manuscript. JJ, SC and FC conceived of the study, and participated in its design and contributed to revisions of the manuscript. LR, WF and ZG participated in experiment materials preparation. All authors read and approved the final manuscript.

\section{Acknowledgments}

This work was supported by the Program for New Century Excellent Talents in University of the Chinese Ministry of Education (Grant No.NCET-10-0492), Fund for Independent Innovation of Agricultural Sciences in Jiangsu Province [CX(12)2020] and the Program for Science and Technology Support, Jiangsu, China (grant no. BE2011325, BE2012350).

Received: 10 July 2014 Accepted: 20 November 2014 Published: 2 December 2014

\section{References}

1. Shinoyama H, Aida R, Ichikawa H, Nomura Y, Mochizuki A: Genetic engineering of chrysanthemum (Chrysanthemum morifolium): current progress and perspectives. Plant Biotechnol 2012, 29(4):323-337.

2. da Silva JA T, Shinoyama H, Aida R, Matsushita Y, Raj SK, Chen F: Chrysanthemum Biotechnology: Quo vadis? Crit Rev Plant Sci 2013, 32(1):21-52. 
3. Kaloshian I, Kinsey M, Ullman D, Williamson V: The impact of Meu1-mediated resistance in tomato on longevity, fecundity and behavior of the potato aphid. Macrosiphum euphorbiae Entomologia Experimentalis et Applicata 1997, 83(2):181-187.

4. Rossi M, Goggin FL, Milligan SB, Kaloshian I, Ullman DE, Williamson VM: The nematode resistance gene $\mathrm{Mi}$ of tomato confers resistance against the potato aphid. Proc Natl Acad Sci 1998, 95(17):9750-9754.

5. Pauquet J, Burget E, Hagen L, Chovelon V, Menn A, Valot N, Desloire S, Caboche M, Rousselle P, Pitrat M: Map-based cloning of the Vat gene from melon conferring resistance to both aphid colonization and aphid transmission of several viruses. In Proceedings of the 8th EUCARPIA Meeting on Cucurbit Genetics and Breeding: 12-17 July 2004. Edited by Lebeda A Olomouc: Paris HS; 2004:325-329.

6. Brotman $Y$, Silberstein L, Kovalski I, Perin C, Dogimont C, Pitrat M, Klingler J, Thompson G, Perl-Treves R: Resistance gene homologues in melon are linked to genetic loci conferring disease and pest resistance. Theor Appl Genet 2002, 104(6-7):1055-1063.

7. Klingler J, Creasy R, Gao L, Nair RM, Calix AS, Jacob HS, Edwards OR, Singh KB: Aphid resistance in Medicago truncatula involves antixenosis and phloem-specific, inducible antibiosis, and maps to a single locus flanked by NBS-LRR resistance gene analogs. Plant Physio/ 2005, 137(4):1445-1455.

8. Smith CM, Boyko EV: The molecular bases of plant resistance and defense responses to aphid feeding: current status. Entomol Exp App/ 2007, 122(1):1-16.

9. Moran PJ, Thompson GA: Molecular responses to aphid feeding in Arabidopsis in relation to plant defense pathways. Plant Physiol 2001, 125(2):1074-1085

10. KUŚNIERCZYK A, Winge P, JØRSTAD TS, TROCZYŃSKA J, Rossiter JT, Bones AM: Towards global understanding of plant defence against aphids-timing and dynamics of early Arabidopsis defence responses to cabbage aphid (Brevicoryne brassicae) attack. Plant Cell Environ 2008, 31(8):1097-1115.

11. Li Q, Xie Q-G, Smith-Becker J, Navarre DA, Kaloshian I: Mi-1-mediated aphid resistance involves salicylic acid and mitogen-activated protein kinase signaling cascades. Mol Plant-Microbe Interact 2006, 19(6):655-664.

12. Kuśnierczyk A, Tran DH, Winge P, Jørstad TS, Reese JC, Troczyńska J, Bones AM: Testing the importance of jasmonate signalling in induction of plant defences upon cabbage aphid (Brevicoryne brassicae) attack. BMC Genomics 2011, 12(1):423.

13. Mewis I, Appel HM, Hom A, Raina R, Schultz JC: Major signaling pathways modulate Arabidopsis glucosinolate accumulation and response to both phloem-feeding and chewing insects. Plant Physiol 2005, 138(2):1149-1162.

14. Adio AM, Casteel CL, De Vos M, Kim JH, Joshi V, Li B, Juéry C, Daron J, Kliebenstein DJ, Jander G: Biosynthesis and defensive function of Nס-acetylornithine, a jasmonate-induced Arabidopsis metabolite. Plant Cell Online 2011, 23(9):3303-3318.

15. Gao L-L, Klingler JP, Anderson JP, Edwards OR, Singh KB: Characterization of pea aphid resistance in Medicago truncatula. Plant Physiol 2008, 146(3):996-1009

16. Argandona V, Chaman M, Cardemil L, Munoz O, Zuniga G, Corcuera L: Ethylene production and peroxidase activity in aphid-infested barley. J Chem Ecol 2001, 27(1):53-68.

17. Liu R, Chen L, Jia Z, Lü B, Shi H, Shao W, Dong H: Transcription factor AtMYB44 regulates induced expression of the ETHYLENE INSENSITIVE2 gene in Arabidopsis responding to a harpin protein. Mol Plant-Microbe Interact 2011, 24(3):377-389.

18. Walling LL: The myriad plant responses to herbivores. J Plant Growth Regul 2000, 19(2):195-216.

19. Moran PJ, Cheng Y, Cassell JL, Thompson GA: Gene expression profiling of Arabidopsis thaliana in compatible plant-aphid interactions. Arch Insect Biochem Physiol 2002, 51(4):182-203.

20. He J, Chen F, Chen S, Lv G, Deng Y, Fang W, Liu Z, Guan Z, He C: Chrysanthemum leaf epidermal surface morphology and antioxidant and defense enzyme activity in response to aphid infestation. J Plant Physiol 2011, 168(7):687-693.

21. Audic S, Claverie J-M: The significance of digital gene expression profiles. Genome Res 1997, 7(10):986-995.

22. Alvarez ME: Salicylic acid in the machinery of hypersensitive cell death and disease resistance. Plant Mol Biol 2000, 44(3):429-442.

23. Aviv DH, Rustérucci C, lii BFH, Dietrich RA, Parker JE, Dangl JL: Runaway cell death, but not basal disease resistance, in $I s d 1$ is SA- and NIM1/NPR1-dependent. Plant J 2002, 29(3):381-391.
24. Pieterse CM, Van Loon L: NPR1: the spider in the web of induced resistance signaling pathways. Curr Opin Plant Biol 2004, 7(4):456-464.

25. Spoel SH, Koornneef A, Claessens SM, Korzelius JP, Van Pelt JA, Mueller MJ, Buchala AJ, Métraux J-P, Brown R, Kazan K: NPR1 modulates cross-talk between salicylate-and jasmonate-dependent defense pathways through a novel function in the cytosol. Plant Cell Online 2003, 15(3):760-770.

26. Kinkema M, Fan W, Dong X: Nuclear localization of NPR1 is required for activation of $P R$ gene expression. Plant Cell Online 2000, 12(12):2339-2350.

27. Howe GA, Jander G: Plant immunity to insect herbivores. Annu Rev Plant Biol 2008, 59:41-66.

28. Koo AJ, Howe GA: The wound hormone jasmonate. Phytochemistry 2009, 70(13):1571-1580.

29. Creelman RA, Mullet JE: Biosynthesis and action of jasmonates in plants. Annu Rev Plant Biol 1997, 48(1):355-381.

30. Voelckel C, Weisser W, Baldwin I: An analysis of plant-aphid interactions by different microarray hybridization strategies. Mol Ecol 2004, 13(10):3187-3195.

31. Fidantsef A, Stout M, Thaler J, Duffey S, Bostock R: Signal interactions in pathogen and insect attack: expression of lipoxygenase, proteinase inhibitor II, and pathogenesis-related protein P4 in the tomato, Lycopersicon esculentum. Physiol Mol Plant Pathol 1999, 54(3):97-114.

32. Kerchev PI, Fenton B, Foyer CH, Hancock RD: Infestation of potato (Solanum tuberosum L.) by the peach-potato aphid (Myzus persicae Sulzer) alters cellular redox status and is influenced by ascorbate. Plant Cell Environ 2012, 35(2):430-440.

33. Chini A, Fonseca S, Fernandez G, Adie B, Chico J, Lorenzo O, Garcia-Casado G, Lopez-Vidriero I, Lozano F, Ponce M: The JAZ family of repressors is the missing link in jasmonate signalling. Nature 2007, 448(7154):666-671.

34. Suza WP, Staswick PE: The role of JAR1 in jasmonoyl-L-isoleucine production during Arabidopsis wound response. Planta 2008, 227(6):1221-1232.

35. Thines B, Katsir L, Melotto M, Niu Y, Mandaokar A, Liu G, Nomura K, He SY, Howe GA, Browse J: JAZ repressor proteins are targets of the SCF ${ }^{\mathrm{CO} 1}$ complex during jasmonate signalling. Nature 2007, 448(7154):661-665.

36. Chung HS, Koo AJ, Gao X, Jayanty S, Thines B, Jones AD, Howe GA: Regulation and function of Arabidopsis JASMONATE ZIM-domain genes in response to wounding and herbivory. Plant Physiol 2008, 146(3):952-964.

37. Pauwels L, Barbero GF, Geerinck J, Tilleman S, Grunewald W, Pérez AC, Chico JM, Bossche RV, Sewell J, Gil E: NINJA connects the co-repressor TOPLESS to jasmonate signalling. Nature 2010, 464(7289):788-791.

38. Divol F, Vilaine F, Thibivilliers S, Amselem J, Palauqui J-C, Kusiak C, Dinant S: Systemic response to aphid infestation by Myzus persicae in the phloem of Apium graveolens. Plant Mol Biol 2005, 57(4):517-540.

39. Boyko EV, Smith CM, Thara VK, Bruno JM, Deng Y, Starkey SR, Klaahsen DL: Molecular basis of plant gene expression during aphid invasion: wheat Pto- and Pti-like sequences are involved in interactions between wheat and Russian wheat aphid (Homoptera: Aphididae). J Econ Entomol 2006, 99(4):1430-1445.

40. Liu R, Lü B, Wang X, Zhang C, Zhang S, Qian J, Chen L, Shi H, Dong H: Thirty-seven transcription factor genes differentially respond to a harpin protein and affect resistance to the green peach aphid in Arabidopsis. J Biosci 2010, 35(3):435-450.

41. Zhu Z, An F, Feng Y, Li P, Xue L, Mu A, Jiang Z, Kim J-M, To TK, Li W: Derepression of ethylene-stabilized transcription factors (EIN3/EIL1) mediates jasmonate and ethylene signaling synergy in Arabidopsis. Proc Natl Acad Sci 2011, 108(30):12539-12544.

42. Pardha Saradhi P, Mohan Ram H: Prolongation of vase-life of chrysanthemum blooms by cobalt chloride and its reversal by IAA. In N International Symposium on Postharvest Physiology of Ornamental Plants 261: 1988; 1988:309-312.

43. Narumi T, Aida R, Ohmiya A, Satoh S: Transformation of chrysanthemum with mutated ethylene receptor genes: $m D G$-ERS1 transgenes conferring reduced ethylene sensitivity and characterization of the transformants. Postharvest Biol Technol 2005, 37(2):101-110.

44. Bodenhausen N, Reymond P: Signaling pathways controlling induced resistance to insect herbivores in Arabidopsis. Mol Plant-Microbe Interact 2007, 20(11):1406-1420.

45. Erb M, Flors V, Karlen D, De Lange E, Planchamp C, D'Alessandro M, Turlings TC, Ton J: Signal signature of aboveground-induced resistance upon belowground herbivory in maize. Plant J 2009, 59(2):292-302.

46. Thaler JS, Bostock RM: Interactions between abscisic-acid-mediated responses and plant resistance to pathogens and insects. Ecology 2004, 85(1):48-58 
47. Erb M, Meldau S, Howe GA: Role of phytohormones in insect-specific plant reactions. Trends Plant Sci 2012, 17(5):250-259.

48. Lorenzo O, Chico JM, Sánchez-Serrano JJ, Solano R: JASMONATEINSENSITIVE1 encodes a MYC transcription factor essential to discriminate between different jasmonate-regulated defense responses in Arabidopsis. Plant Cell Online 2004, 16(7):1938-1950.

49. Dombrecht B, Xue GP, Sprague SJ, Kirkegaard JA, Ross JJ, Reid JB, Fitt GP, Sewelam N, Schenk PM, Manners JM: MYC2 differentially modulates diverse jasmonate-dependent functions in Arabidopsis. Plant Cell Online 2007, 19(7):2225-2245.

50. Grunewald W, Vanholme B, Pauwels L, Plovie E, Inzé D, Gheysen G, Goossens A: Expression of the Arabidopsis jasmonate signalling repressor JAZ1/TIFY10A is stimulated by auxin. EMBO Rep 2009, 10(8):923-928.

51. Tiryaki I, Staswick PE: An Arabidopsis mutant defective in jasmonate response is allelic to the auxin-signaling mutant axr1. Plant Physiol 2002, 130(2):887-894.

52. Hou X, Lee LYC, Xia K, Yan Y, Yu H: DELLAs modulate jasmonate signaling via competitive binding to JAZs. Dev Cell 2010, 19(6):884-894.

53. Wang $H$, Hao J, Chen $X$, Hao Z, Wang X, Lou Y, Peng Y, Guo Z: Overexpression of rice WRKY89 enhances ultraviolet $B$ tolerance and disease resistance in rice plants. Plant Mol Biol 2007, 65(6):799-815.

54. Skibbe M, Qu N, Galis I, Baldwin IT: Induced plant defenses in the natural environment: Nicotiana attenuata WRKY3 and WRKY6 coordinate responses to herbivory. Plant Cell Online 2008, 20(7):1984-2000.

55. Lu J, Ju H, Zhou G, Zhu C, Erb M, Wang X, Wang P, Lou Y: An EAR-motif-containing ERF transcription factor affects herbivore-induced signaling, defense and resistance in rice. Plant J 2011, 68(4):583-596.

56. Mittler R, Vanderauwera S, Suzuki N, Miller G, Tognetti VB, Vandepoele K, Gollery M, Shulaev V, Van Breusegem F: ROS signaling: the new wave? Trends Plant Sci 2011, 16(6):300-309.

57. Marino D, Dunand C, Puppo A, Pauly N: A burst of plant NADPH oxidases. Trends Plant Sci 2012, 17(1):9-15.

58. Pennell RI, Lamb C: Programmed cell death in plants. Plant Cell 1997, 9(7):1157.

59. Moloi MJ, van der Westhuizen AJ: The reactive oxygen species are involved in resistance responses of wheat to the Russian wheat aphid. J Plant Physiol 2006, 163(11):1118-1125.

60. Ushio-Fukai M: Localizing NADPH oxidase-derived ROS. Sci Signal 2006, 2006(349):re8.

61. Passardi F, Penel C, Dunand C: Performing the paradoxical: how plant peroxidases modify the cell wall. Trends Plant Sci 2004, 9(11):534-540.

62. Zhu-Salzman K, Salzman RA, Ahn J-E, Koiwa H: Transcriptional regulation of sorghum defense determinants against a phloem-feeding aphid. Plant Physiol 2004, 134(1):420-431.

63. Dogimont C, Chovelon V, Tual S, Boissot N, Rittener-Rüff V, Giovinazzo N Bendahmane A, Pitrat M: Molecular Diversity at the Vat/Pm-W Resistance Locus in Melon; 2008

64. Botha A, Lacock L, Van Niekerk C, Matsioloko M, De Preez F, Myburg A, Proceedings of 10th International Wheat Genetic Symposium: 2003: Gene Expression Profiling During Diuraphis Noxia Infestation of Triticum Aestivum cv. 'Tugela DN' Using Microarrays. In Proceedings of 10th International Wheat Genetic Symposium; 2003:334-338.

65. Lacock L, Botha A: Suppression Subtractive Hybridization (SSH) Employed to Investigate Gene Expression After Russian Wheat Aphid Infestation. In Proceedings of the 10th International Wheat Genetics Symposium, Paestum, Italy (1-6 September 2003): 2003; 2003:1187-1189.

66. Van Niekerk C, Botha A: Using Suppression Subtractive Hybridization (SSH) to Screen for Novel Sequences Expressed in Response to Russian Wheat Aphid Feeding. In Proceedings of the 10th International Wheat Genetics Symposium, Paestum, Italy (1-6 September 2003): 2003; 2003:1281-1283

67. Swanepoel E, Lacock L, Myburg A, Botha A: A Leucine Rich Homolog to Aegilops Tauschii from Bread Wheat Line PI137739 Obtained by Suppression Subtractive Hybridization Shows Linkage to Russian Wheat Aphid Resistance Gene Dn1. In Proceedings of the 10th International Wheat Genetics Symposium, Paestum, Italy (1-6 September 2003): 2003; 2003:1263-1265.

68. Schindelman G, Morikami A, Jung J, Baskin TI, Carpita NC, Derbyshire P, McCann MC, Benfey PN: COBRA encodes a putative GPI-anchored protein, which is polarly localized and necessary for oriented cell expansion in Arabidopsis. Genes Dev 2001, 15(9):1115-1127.
69. Hazen SP, Scott-Craig JS, Walton JD: Cellulose synthase-like (CSL) genes of rice. Plant Physiol 2002, 128:336-340.

70. Li Y, Qian Q, Zhou Y, Yan M, Sun L, Zhang M, Fu Z, Wang Y, Han B, Pang X: BRITTLE CULM1, which encodes a COBRA-like protein, affects the mechanical properties of rice plants. Plant Cell Online 2003, 15(9):2020-2031.

71. Holland N, Holland D, Helentjaris T, Dhugga KS, Xoconostle-Cazares B, Delmer DP: A comparative analysis of the plant cellulose synthase (CesA) gene family. Plant Physiol 2000, 123(4):1313-1324.

72. Joshi CP, Mansfield SD: The cellulose paradox-simple molecule, complex biosynthesis. Curr Opin Plant Biol 2007, 10(3):220-226.

73. Richmond TA, Somerville CR: The cellulose synthase superfamily. Plant Physiol 2000, 124(2):495-498.

74. Leszczynski B, Wright LC, Bakowski T: Effect of secondary plant-substances on winter-wheat resistance to grain aphid. Entomol Exp Appl 1989, 52(2):135-139.

75. Lattanzio V, Arpaia S, Cardinali A, Di Venere D, Linsalata V: Role of endogenous flavonoids in resistance mechanism of Vigna to aphids. J Agric Food Chem 2000, 48(11):5316-5320.

76. Onkokesung N, Reichelt M, van Doorn A, Schuurink RC, van Loon JJA, Dicke M: Modulation of flavonoid metabolites in Arabidopsis thaliana through overexpression of the MYB75 transcription factor: role of kaempferol-3,7dirhamnoside in resistance to the specialist insect herbivore Pieris brassicae. J Exp Bot 2014, 65(8):2203-2217.

77. Treutter D: Significance of flavonoids in plant resistance: a review. Environ Chem Lett 2006, 4(3):147-157.

78. Schnee C, Kollner TG, Gershenzon J, Degenhardt J: The maize gene terpene synthase 1 encodes a sesquiterpene synthase catalyzing the formation of (E)-beta-farnesene, (E)-nerolidol, and (E, E)-farnesol after herbivore damage. Plant Physiol 2002, 130(4):2049-2060.

79. Deng Y, Chen S, Lu A, Chen F, Tang F, Guan Z, Teng N: Production and characterisation of the intergeneric hybrids between Dendranthema morifolium and Artemisia vulgaris exhibiting enhanced resistance to chrysanthemum aphid (Macrosiphoniella sanbourni). Planta 2010, 231(3):693-703.

80. Miles PW: Aphid saliva. Biol Rev 1999, 74(1):41-85.

doi:10.1186/1471-2164-15-1050

Cite this article as: Xia et al.: Gene expression profiles responses to aphid feeding in chrysanthemum (Chrysanthemum morifolium). BMC Genomics 2014 15:1050.

\section{Submit your next manuscript to BioMed Central and take full advantage of:}

- Convenient online submission

- Thorough peer review

- No space constraints or color figure charges

- Immediate publication on acceptance

- Inclusion in PubMed, CAS, Scopus and Google Scholar

- Research which is freely available for redistribution 